\title{
Article \\ The Effect of Selected Herbal Extracts on Lactic Acid Bacteria Activity
}

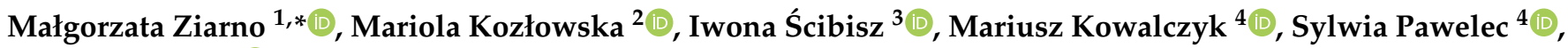 \\ Anna Stochmal ${ }^{4}$ (D) and Bartłomiej Szleszyński 5
}

1 Division of Milk Technology, Department of Food Technology and Assessment, Institute of Food Science, Warsaw University of Life Sciences-SGGW (WULS-SGGW), 02-787 Warsaw, Poland

2 Department of Chemistry, Institute of Food Science, Warsaw University of Life Sciences-SGGW (WULS-SGGW), 02-787 Warsaw, Poland; mariola_kozlowska@sggw.edu.pl

3 Division of Fruit, Vegetable and Cereal Technology, Department of Food Technology and Assessment, Institute of Food Science, Warsaw University of Life Sciences-SGGW (WULS-SGGW), 02-787 Warsaw, Poland; iwona_scibisz@sggw.edu.pl

4 Department of Biochemistry and Crop Quality, Institute of Soil Science and Plant Cultivation, State Research Institute, 24-100 Puławy, Poland; mkowalczyk@iung.pulawy.pl (M.K.); spawelec@iung.pulawy.pl (S.P.); asf@iung.pulawy.pl (A.S.)

5 Institute of Horticultural Sciences, Warsaw University of Life Sciences-SGGW (WULS-SGGW), 02-787 Warsaw, Poland; bartekszleszynski@gmail.com

* Correspondence: malgorzata_ziarno@sggw.edu.pl; Tel.: +48-225-937-666

Citation: Ziarno, M.; Kozłowska, M.; Ścibisz, I.; Kowalczyk, M.; Pawelec, S.; Stochmal, A.; Szleszyński, B. The Effect of Selected Herbal Extracts on Lactic Acid Bacteria Activity. Appl. Sci. 2021, 11, 3898. https://doi.org/ 10.3390/app11093898

Academic Editor:

Gwiazdowska Daniela

Received: 1 April 2021

Accepted: 21 April 2021

Published: 25 April 2021

Publisher's Note: MDPI stays neutral with regard to jurisdictional claims in published maps and institutional affiliations.

Copyright: (c) 2021 by the authors. Licensee MDPI, Basel, Switzerland. This article is an open access article distributed under the terms and conditions of the Creative Commons Attribution (CC BY) license (https:// creativecommons.org/licenses/by/ $4.0 /)$.

\begin{abstract}
This study aimed to investigate the effect of plant extracts (valerian Valeriana officinalis L., sage Salvia officinalis L., chamomile Matricaria chamomilla L., cistus Cistus L., linden blossom Tilia L., ribwort plantain Plantago lanceolata L., marshmallow Althaea L.) on the activity and growth of lactic acid bacteria (LAB) during the fermentation and passage of milk through a digestive system model. The tested extracts were also characterized in terms of their content of polyphenolic compounds and antioxidant activity. It was observed that the addition of the tested herbal extracts did not inhibit the growth of LAB in fermented milk drinks, such as yogurts. However, they can gradually inhibit fermentation when added at concentrations above $2 \%$ by weight, and hence should be used in limited amounts. The microflora of yogurts containing the tested herbal extracts did not die during digestion in model digestive juices, and no stimulating effect of the added plant extracts was noted either. Nevertheless, due to the antioxidant properties, a slight addition of the herbal extracts containing polyphenols to different kinds of food products can increase the nutritional quality, thus making them functional foods.
\end{abstract}

Keywords: lactic acid bacteria; plant extracts; milk fermentation; total phenolic content; antioxidant capacities

\section{Introduction}

The benefits of fermented milk drinks seem to be recognized by consumers as the consumption of these products continues to increase, particularly in industrialized countries. In recent years, manufacturers have been outdoing each other in inventing novel products to attract customers. For instance, yogurts are prepared with various fruits, which are often quite exotic and rarely found in local markets. It has been found that plant extracts that were used in folk medicine and sometimes even in culinary applications may serve as interesting additives. However, the effect of their addition on the microflora of fermented milk beverages should be investigated.

Valerian (Valeriana officinalis L.) has been a known herb since ancient Greek and Roman times. The action of this herb was described by Dioscorides, Hippocrates, and Plinius Secundus. Valerian is one of the most popular herbs used in various medications, mainly for its calming and relaxing effects. In folk medicine, it was used as a sedative and as a relaxant 
of the smooth muscles of the digestive tract, urinary tract, and blood vessels. The name of the herb is derived from the Latin word "valere", which means "to be healthy" [1,2]. Sage (Salvia officinalis L.) contains compounds that inhibit lipid peroxidation, and also exhibit antibacterial, antiallergic, antiviral, and analgesic properties. In addition, sage is used in medicine to fight rheumatism and arthritis, and sometimes to prevent the general weakening of the body [3-5]. Chamomile (Matricaria chamomilla L.) has been used for centuries as a poultice for healing wounds and burns, and as an eyewash to treat conjunctivitis. This herb has not lost its relevance [6-8]. It is known to have anti-inflammatory, antibacterial, bacterial toxin-binding, disinfectant, antispasmodic, choleretic, sedative, and laxative effects, and is hence used widely in medicine. Furthermore, it is used in the food industry for the production of liqueurs and in the cosmetic industry for the production of creams, lotions, soaps, bath liquids, and toothpastes [6-8]. Cistus (Cistus L.) acts as an antioxidant and removes free radicals and prevents the formation of new ones $[9,10]$; it also has antibacterial, anti-inflammatory, antiviral, and antifungal effects [9,10]. Linden blossom (Tilia L.) exhibits a high healing effect and was therefore used by our ancestors for generations. In traditional medicine, the linden flower is commonly used for nervous tension and excessive nervous excitability. In some cases, it is used as a prophylactic against atherosclerosis and hypertension [11-16]. Linden flowers are also characterized by anti-inflammatory, antipyretic, diaphoretic, diuretic, mildly astringent, and analgesic properties [11-16]. Since ancient times, infusions prepared from linden or sage have been used to treat respiratory diseases. Ribwort plantain (Plantago lanceolata L.) has also been used in folk medicine. Its leaves are used to treat cuts, bruises, bites, and burns, as well as for chronic gastrointestinal catarrh, acidity, and damage to the gastric and intestinal mucosa (e.g., by bacterial toxins or other chemical compounds) [17-20]. This herb has bacteriostatic, antimicrobial, anti-inflammatory, antispasmodic, and expectorant properties. It also improves blood clotting and has astringent and sealing effects on blood vessels [17-20]. Marshmallow (Althaea L.) was valued for its prohealth properties by Egyptians and Syrians in ancient times. The generic name Althaea comes from the Greek word "althe" which means "to heal". Both the root and leaves of marshmallow are rich in mucus, which is the basic ingredient of this plant used in medicine [21-24]. Marshmallow has anti-inflammatory, protective, coating, and antitussive properties [21-24].

In food production, the use of herbs can stabilize the microflora of products, by preventing the development of harmful microorganisms and supporting the growth of desired bacteria. Only few scientific reports have shown that when selected plant extracts are used in appropriate portions, they can have a beneficial effect on lactic acid bacteria (LAB) $[25,26]$. Bifidobacteria and some lactobacilli can transform polyphenols into important metabolites that have important functions in the human body is known to be [25,26]. Moreover, observations of market trends suggest that plant extracts are used or can be used as food additives in the production of flavored fermented dairy products (including yoghurts or other fermented milks, sour cream, acid and rennet cheeses), and many products derived from them. Only limited studies have analyzed the influence of phenolic compounds on the growth and viability of other lactic acid bacteria such as Streptococcus thermophilus used in the production of yogurt.

The present study aimed to investigate the effect of selected herbal additives on the activity and growth of lactic acid bacteria (LAB) during fermentation and passage of milk through the digestive system model. Additionally, the tested extracts were characterized in terms of their content of polyphenolic compounds and antioxidant activity.

\section{Materials and Methods}

\subsection{Materials}

The following plant extracts were used in the research: valerian ( $V$. officinalis L.), sage (S. officinalis L.), chamomile (M. chamomilla L.), cistus (Cistus L.), linden blossom (Tilia L.), ribwort plantain (P. lanceolata L.), and marshmallow (Althaea L.). They were purchased from GreenVit sp. z.o.o. (Zambrów, Poland). These were water extracts 
obtained by percolation at elevated temperatures. Then, they were concentrated in a vacuum evaporator, and possibly dried further under vacuum. Marshmallow and plantain extracts were obtained in liquid form, while the rest were in powder form. Maltodextrin was used as the drying carrier.

\subsection{Determination of the Effect of Plant Extracts on the Lactic Acid Fermentation of Milk}

In the first stage of the work, the ability of LAB to ferment milk was checked in the presence of selected plant extracts. Briefly, the milk samples intended for lactic acid fermentation was prepared from UHT milk (containing 3.2\% of fat) in a volume of $100 \mathrm{~mL}$, and the plant extracts were added at amounts of $0.2,0.6,1.0,1.4,2.0,3.0,4.0$, and 5.0\%. Then, the samples were transferred to a water bath heated to $42{ }^{\circ} \mathrm{C}$, and the starter culture was added (at an amount of $0.04 \%$ ). The yogurt starter culture YC-X16 (received kindly from Chr. Hansen Poland) was used in the research. This freeze-dried culture is composed of Streptococcus thermophilus and Lactobacillus delbrueckii subsp. bulgaricus. Fermentation of milk samples was carried out at $42^{\circ} \mathrm{C}$. For the next $4 \mathrm{~h}$, the $\mathrm{pH}$ of the samples was measured every 30 min until the end of fermentation, using a standard laboratory stationary $\mathrm{pH}$ meter with three replications.

\subsection{Determination of the Effect of Plant Extracts on the Populations of Lactic Acid Bacteria}

The effect of plant extracts on the population of LAB cells was investigated immediately after the fermentation process and digestion of fermented milk in model digestive juices. At this stage, however, based on the results of the first stage, only the following portions of plant extracts were used: $0.2,0.6,1.0$, and $1.4 \%$ extracts added to UHT milk. Fermentation was carried out as in the first stage of the research. After the end of the process, the number of LAB cells in the samples was determined. M17 (MERCK) and MRS agar (De Man Rogosa Sharpe Agar, MERCK) were used in the analysis. The inoculated Petri plates were incubated in an incubator at $37^{\circ} \mathrm{C}$ under aerobic (M17 agar) or anaerobic (MRS agar) condition. The cell count was determined after $72 \mathrm{~h}$, and the results are expressed in colony forming units in $1 \mathrm{~mL}$ of the sample $(\mathrm{CFU} / \mathrm{mL})$.

The next stage of the work consisted of several steps. The first step involved the digestion of the fermented milk samples added with plant extracts under gastric juice conditions, and the second step involved the digestion of the samples under intestinal juice conditions [27]. Gastric juice was prepared as described by Ziarno and Zareba [27]. Briefly, $4.8 \mathrm{~g}$ of $\mathrm{NaCl}, 1.56 \mathrm{~g}$ of $\mathrm{NaHCO}_{3}, 2.2 \mathrm{~g}$ of $\mathrm{KCl}$, and $0.22 \mathrm{~g}$ of $\mathrm{CaCl}_{2}$ were dissolved in $1000 \mathrm{~mL}$ of distilled water. The $\mathrm{pH}$ of the prepared solution was adjusted to 2.40 with $1 \mathrm{M}$ $\mathrm{HCl}$. Then, the solution was sterilized in an autoclave at $121^{\circ} \mathrm{C}$ for $15 \mathrm{~min}$. Immediately before the experiment, pepsin (Sigma-Aldrich) was added to the solution at an amount of $285 \mu \mathrm{L} / 100 \mathrm{~mL}$ gastric juice. The model intestinal juice was also prepared as described by Ziarno and Zareba [27]. Briefly, $5 \mathrm{~g}$ of $\mathrm{NaCl}, 0.6 \mathrm{~g}$ of $\mathrm{KCl}, 0.25 \mathrm{~g}$ of $\mathrm{CaCl}_{2}$, and $8.5 \mathrm{~g}$ of beef bile were dissolved respectively in $1 \mathrm{M} \mathrm{NaHCO}_{3}$. The $\mathrm{pH}$ of the prepared solution was adjusted to 7.0 with $1 \mathrm{M} \mathrm{HCl}$. The whole mixture was successively sterilized in an autoclave at $121^{\circ} \mathrm{C}$ for $15 \mathrm{~min}$. Immediately before the experiment, one Kreon Travix 10,000 capsule (Abbott Laboratories) was added to $200 \mathrm{~mL}$ of model intestinal juice. The capsule contains a mixture of digestive enzymes, which at a dose of $150 \mathrm{mg}$ shows the activity of 10,000 IU Ph. Eur lipase, 8000 units Ph. Eur amylase, and 600 Ph. Eur proteases. Digestion was carried out in a static system by mixing $35 \mathrm{~mL}$ of model gastric juice with the appropriate amount of pepsin and $35 \mathrm{~mL}$ of fermented milk sample. Gastric juice digestion was performed for $3 \mathrm{~h}$ at $37^{\circ} \mathrm{C}$, and then the mixture was transferred to the same amount of model intestinal juice for digestion which lasted for $5 \mathrm{~h}$ at $37^{\circ} \mathrm{C}$. Finally, the number of viable LAB cells was determined as described above.

\subsection{Determination of TPC of Plant Extracts}

Total phenolic content (TPC) was estimated in the plant extracts using the FolinCiocalteu method as described previously [28] with a slight modification. First, appro- 
priately diluted plant extract $(3 \mathrm{mg} / \mathrm{mL})$ was mixed with deionized water $(20 \mathrm{~mL})$ and Folin-Ciocalteu reagent $(0.5 \mathrm{~mL})$. After $30 \mathrm{~s}, 5 \mathrm{~mL}$ of $\mathrm{Na}_{2} \mathrm{CO}_{3}(20 \%, v / v)$ was added to the solution. Then, the solution was incubated at $21^{\circ} \mathrm{C}$ for $1 \mathrm{~h}$, and its absorbance was measured at $765 \mathrm{~nm}$ using a UV/Vis spectrophotometer (Model 8500; Techcomp, Hong Kong). The results were expressed as mg gallic acid equivalents per gram of extract (mg GAE/g extract) using a standard gallic acid calibration curve. The analysis was performed in three independent replications.

\subsection{Determination of Antioxidant Capacities of Plant Extracts}

Before the analysis of antioxidant activity, each extract (3-6 mg) was diluted with distilled water (2-4 mL). The plant extract solutions were thus prepared in triplicate, and their average values of antioxidant capacity were determined as mmol Trolox equivalents per gram of extract (mmol TE/g extract).

DPPH (2,2-diphenyl-1-picrylhydrazyl) radical scavenging activity was performed according to a procedure described by Yen and Chen [29] with minor modification. Briefly, $1 \mathrm{~mL}$ of $0.3 \mathrm{mmol} / \mathrm{L}$ freshly prepared DPPH methanol solution was mixed with $0.2 \mathrm{~mL}$ of the plant extract solution and $3.8 \mathrm{~mL}$ of methanol. The samples were vortex-mixed at high speed for $10 \mathrm{~s}$ and incubated for $10 \mathrm{~min}$ in the dark at room temperature. Then, their absorbance was measured at $517 \mathrm{~nm}$ using a UV/Vis spectrophotometer. A standard curve was obtained using the Trolox standard in the range of 8-40 $\mu \mathrm{mol} / \mathrm{L}$.

ABTS (2,2'-azinobis(3-ethylbenthiazoline-6-sulfonic acid)) radical scavenging activity of the extracts was determined according to the method described by Re et al. [30]. First, the ABTS•+ solution was prepared by mixing ABTS aqueous solution $(14 \mathrm{mmol} / \mathrm{L})$ with potassium persulfate aqueous solution $(4.9 \mathrm{mmol} / \mathrm{L})$. The prepared solution was kept for 12-16 $\mathrm{h}$ in the dark at room temperature. Before the analysis, the ABTS•+ solution was diluted with phosphate-buffered saline $(0.01 \mathrm{~mol} / \mathrm{L}, \mathrm{pH} 7.4)$ to achieve an absorbance value of $0.7 \pm 0.05$ at $734 \mathrm{~nm}$. Then, $40 \mu \mathrm{L}$ of the plant extract solution or Trolox solution was mixed with $4 \mathrm{~mL}$ of ABTS $\bullet+$ working solution. The reactive mixture was incubated at room temperature in the dark, and after exactly $6 \mathrm{~min}$, its absorbance was recorded at $734 \mathrm{~nm}$. A series of Trolox solutions (final concentrations 0-15 $\mu \mathrm{M}$ ) were used for calibration.

FRAP (ferric reducing antioxidant power) assay was performed as described by Benzie and Strain [31] with some modification. Before the assay, the FRAP reagent was freshly prepared by mixing $300 \mathrm{mmol} / \mathrm{L}$ acetate buffer ( $\mathrm{pH} 3.6), 20 \mathrm{mmol} / \mathrm{L} \mathrm{FeCl3}$ solution, and $10 \mathrm{mmol} / \mathrm{L}$ TPTZ (2,4,6-Tris(2-pyridyl)-s-triazine) in $40 \mathrm{mmol} / \mathrm{L} \mathrm{HCl}$ in a 10:1:1 (v/v/v) proportion and stored away from light. Then, $100 \mu \mathrm{L}$ of the appropriately diluted sample extract was mixed with $0.3 \mathrm{~mL}$ distilled water and $3 \mathrm{~mL}$ FRAP reagent. The absorbance of the reaction mixture was measured spectrophotometrically at $593 \mathrm{~nm}$ after incubation at $37^{\circ} \mathrm{C}$ for $10 \mathrm{~min}$. The blank solution was obtained by mixing $0.3 \mathrm{~mL}$ distilled water with $3 \mathrm{~mL}$ of FRAP reagent. A standard curve was prepared using Trolox in the range of 80-500 $\mu \mathrm{mol} / \mathrm{L}$. All determinations were carried out in triplicate.

\subsection{ESI-QTOF Qualitative Analysis of Plant Extracts}

High-resolution liquid chromatography (LC)-mass spectrometry (MS) analyses (exact masses, MS/MS fragmentation patterns, molecular formulas) were performed on a Thermo Scientific Ultimate 3000 RS chromatographic system coupled with a Bruker Impact II HD (Bruker, Billerica, MA, USA) quadrupole time-of-flight (QTOF) mass spectrometer. Chromatographic separations were carried out on a Waters BEH C18 column $(2.1 \times 150 \mathrm{~mm}$, $1.7 \mu \mathrm{m}$; Milford, MA USA), equipped with precolumn. Mobile phase A used was $0.1 \%(\mathrm{v} / \mathrm{v})$ formic acid, while mobile phase B was acetonitrile containing $0.1 \%(\mathrm{v} / \mathrm{v})$ of formic acid. A gradient from 7 to $80 \%$ of phase B over 30 min was used for separation. The flow rate was set at $0.5 \mathrm{~mL} / \mathrm{min}$, and the column was held at a temperature of $60{ }^{\circ} \mathrm{C}$. The injection volume was $5 \mu \mathrm{L}$. The light absorption patterns of the investigated sample components were obtained in the wavelength range of 190-600 nm using a photodiode array detector (Thermo Ultimate DAD-3000) with an analytical flow cell. The column's effluent was split 
into 1:3 proportions between the two detectors operating in parallel, the mass spectrometer, and the charged aerosol detector (CAD), to identify the main constituents of the investigated samples. For identification, data were collected from the mass spectral analyses in both positive and negative ion modes with electrospray ionization (ESI). Linear (centroid) mass spectra were acquired over a mass range from m/z 50 to 2000 with the following MS parameters: positive ion capillary voltage, $4.5 \mathrm{kV}$; negative ion capillary voltage, $3.0 \mathrm{kV}$, dry gas (N2) flow, $6 \mathrm{~L} / \mathrm{min}$; dry gas temperature, $200{ }^{\circ} \mathrm{C}$; and nebulizer gas (N2) pressure, 0.7 bar. Argon was used as the collision gas. The MS/MS collision energy and parent mass isolation width were automatically set between 2.5 and $35 \mathrm{eV}$ and between 2 and 6 mass units, depending on the $\mathrm{m} / \mathrm{z}$ of the fragmented ion. The parameters for ion transfer were optimized for m/z 50-2000, with collision cell transfer time at $80 \mu$ s and prepulse storage at $10 \mu \mathrm{s}$. The acquired data were calibrated internally with $10 \mathrm{mM}$ sodium formate introduced to the ion source via a $20-\mu \mathrm{L}$ loop at the beginning of each separation process. Data processing was carried out using Bruker DataAnalysis 4.3 software. The main components of the sample were identified from the CAD peak areas, while the constituents were identified based on the light absorption properties, precise mass measurements (measurement error $<5 \mathrm{ppm}$ ) of the primary ion $\mathrm{m} / \mathrm{z}$, which allowed for calculating the molecular formula, and software-aided analysis of the isotopic and MS/MS fragmentation patterns [32].

\subsection{Statistical Analysis}

Multifactor analysis of variance (ANOVA) is used in statistical analysis to determine the influence of significant factors in a multivariate model. This is a typical system used for experimental analysis which, in addition to checking the influence of one factor, allows checking the interaction of individual factors with each other. In this study, in conjunction with ANOVA, Tukey's test was used at a significance level of 0.05 to analyze the mutual influence of two factors on each other and find those that differ significantly from each other, which shows the pairs that are statistically significant.

\section{Results}

\subsection{Determination of the Effect of Plant Extracts on Lactic Acid Fermentation}

In the first stage of the research, changes in the $\mathrm{pH}$ of milk samples enriched with portions of plant extracts were analyzed. The obtained results are presented in Figure 1. The change in $\mathrm{pH}$ during the fermentation process indicated that none of the added plant extracts inhibited fermentation. Statistical analysis showed that only the milk samples added with the first three doses of the extracts (from 0 to $1.0 \%$ ) constituted one homogeneous group $(p=0.001)$, where the $\mathrm{pH}$ values were higher by on average $0.2-0.3$ than the samples added with higher doses of the tested plant extracts. The milk samples with $1.0 \%$ or higher portion of the plant extracts constituted separate homogeneous groups $(p=0.001)$ for each analyzed extract. It should be noted that the $\mathrm{pH}$ did not reduce below 4.5 for the addition of any extract above $2.0 \%$ concentration. For selected additives (sage and cistus), a $\mathrm{pH}$ of even 4.1 was achieved for the lowest dose, and 4.8 for $5.0 \%$ dose. For the remaining additives, the differences in $\mathrm{pH}$ observed between their lowest and the highest portion were approximately 0.4 . This difference is significant and confirms that a large amount of additives can inhibit the biochemical activity (i.e., acidifying activity) of LAB present in the yogurt starter culture. In all cases of milk samples fermented in the presence of the tested plant extracts, the $\mathrm{pH}$ was no longer statistically significantly reduced after $3.5 \mathrm{~h}$ of fermentation. 


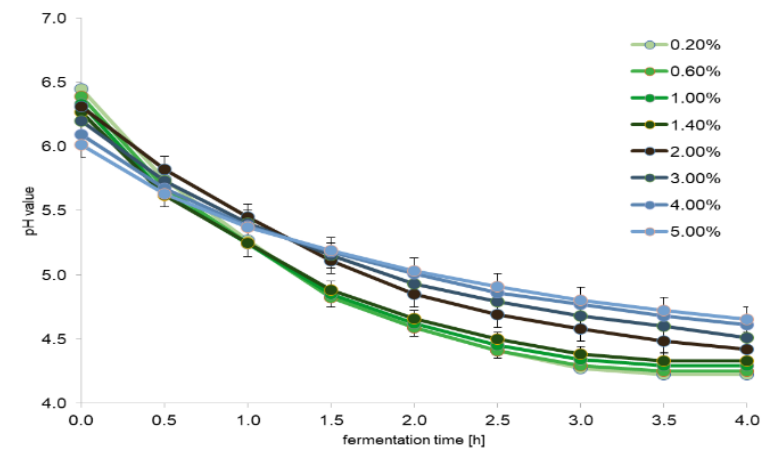

(a)

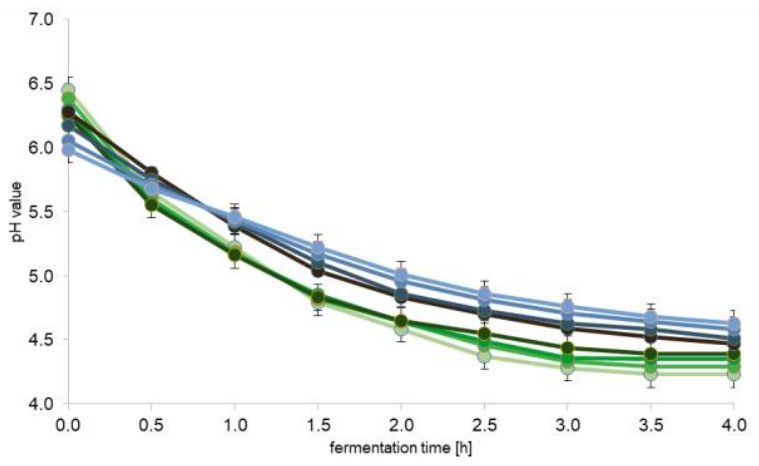

(c)

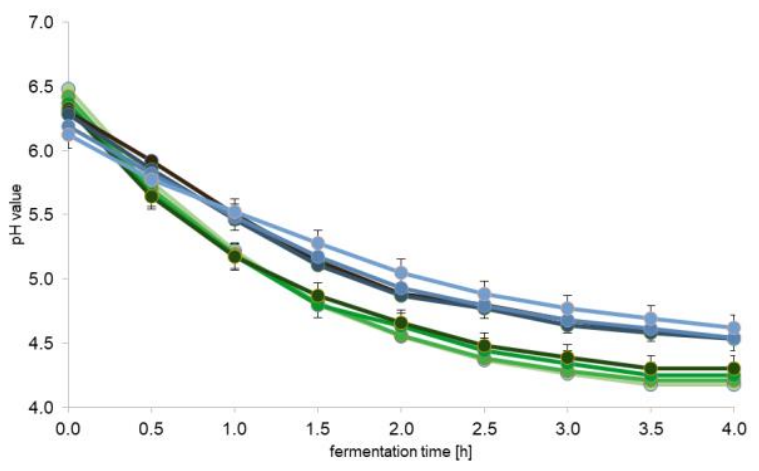

(e)

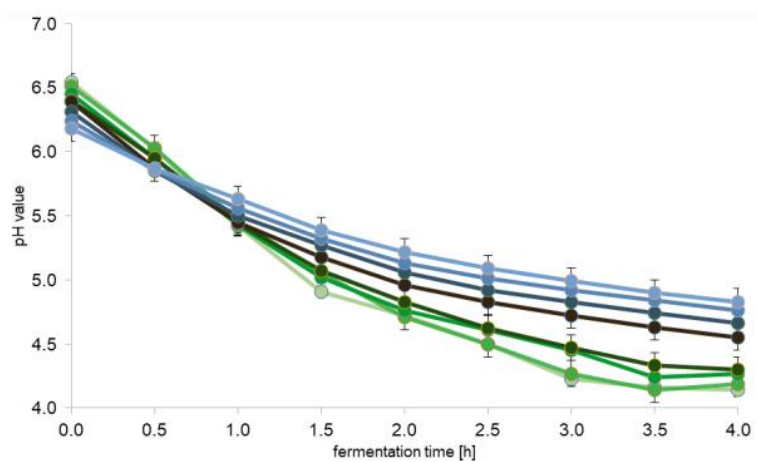

(b)

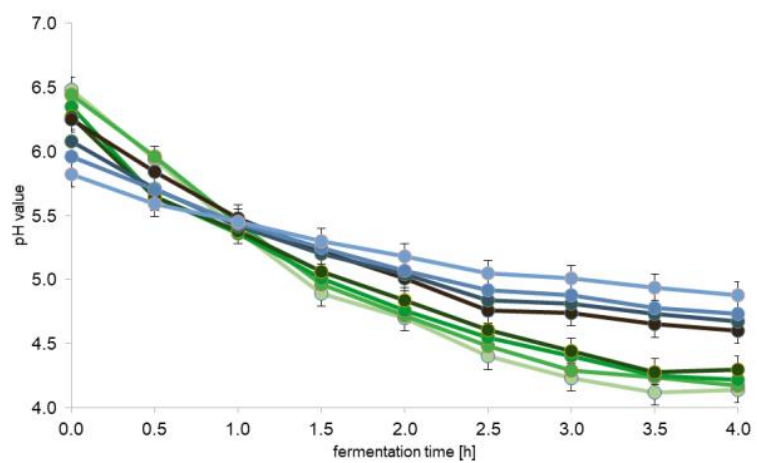

(d)



(f)

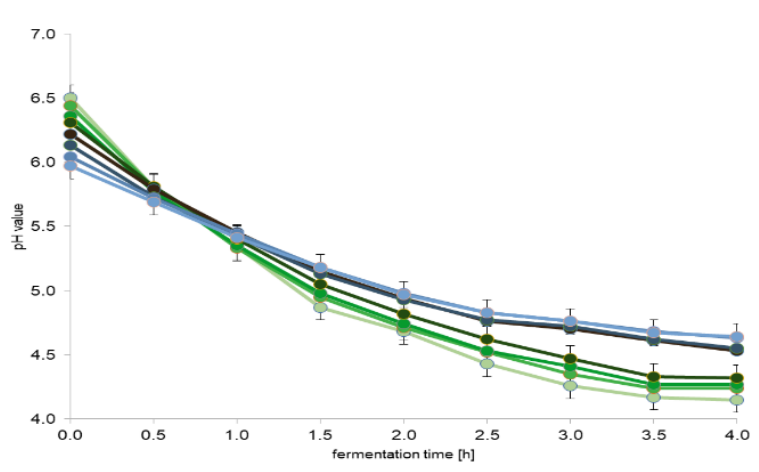

(g)

Figure 1. The effect of plant extracts on lactic acid fermentation (average values and standard deviations of three replicates): (a) valerian (Valeriana officinalis L.); (b) sage (Salvia officinalis L.); (c) chamomile (Matricaria chamomilla L.); (d) cistus (Cistus L.); (e) linden blossom (Tilia L.); (f) ribwort plantain (Plantago lanceolata L.); and (g) marshmallow (Althaea L.). 


\subsection{Determination of the Effect of Plant Extracts on the Populations of Lactic Acid Bacteria}

The results of the determination of both tested LAB populations (Table 1) immediately after the end of the fermentation process confirmed that most of the tested plant extracts did not reduce the populations $(p<0.05)$. This means that the tested LAB cultures well tolerated the presence of the extracts at an amount of up to $3.0 \%$ in milk. Only with the addition of sage extract, we observed some slight, but statistically significant, reduction in the Lactobacillus cell population (Table 1).

Further analyses showed that the types of additives and the digestion process had an impact on the population of $S$. thermophilus cells $(p<0.05)$; however, no effect on the lactobacilli population was observed $(p>0.05)$. It should be noted that both bacterial species had different survival rates in the samples and reacted differently to digestion in the model digestive juices (Table 1). Statistical analysis carried out for individual bacterial species showed that only digestion influenced the number of $S$. thermophilus cells in most cases. The exceptions were the milk samples fermented with the addition of cistus extract and marshmallow extract, in which the digestion step did not statistically significantly influence the $S$. thermophilus cell population. This may suggest that these two extracts improved the survival of $S$. thermophilus cells under the conditions of the model digestive system. In the case of Lactobacillus bacteria, neither the type of plant extract or its dose nor digestion in the model digestive system had a statistically significant influence on the bacterial cell population (Table 1). 
Table 1. The effect of plant extracts on the populations of lactic acid bacteria (average values and standard deviations of five replicates).

\begin{tabular}{|c|c|c|c|c|c|c|c|c|c|c|c|c|c|}
\hline \multirow{3}{*}{ Plant Extracts } & \multirow{3}{*}{$\begin{array}{l}\text { Sampling } \\
\text { Time }\end{array}$} & \multicolumn{6}{|c|}{ Additive Level } & \multicolumn{6}{|c|}{ Additive Level } \\
\hline & & $0.2 \%$ & $0.6 \%$ & $1.0 \%$ & $1.4 \%$ & $2.0 \%$ & $3.0 \%$ & $0.2 \%$ & $0.6 \%$ & $1.0 \%$ & $1.4 \%$ & $2.0 \%$ & $3.0 \%$ \\
\hline & & & Stre & $\begin{array}{r}\text { tococcus thern } \\
{[\log C}\end{array}$ & $\begin{array}{l}\text { ophilus Popul } \\
\text { U/mL] }\end{array}$ & & & & Lactobacill & $\begin{array}{r}\text { delbrueckii su } \\
{[\log \mathrm{CF}}\end{array}$ & $\begin{array}{l}\text { sp. bulgaricu } \\
\mathrm{J} / \mathrm{mL}]\end{array}$ & Population & \\
\hline \multirow{2}{*}{$\begin{array}{l}\text { Valerian } \\
\text { (Valeriana } \\
\text { officinalis L.) }\end{array}$} & \multirow{2}{*}{$\begin{array}{l}\text { after fer- } \\
\text { mentation } \\
\text { after } \\
\text { digestion }\end{array}$} & $7.9 \pm 0.3^{a}$ & $7.6 \pm 0.5^{\mathrm{a}}$ & $7.8 \pm 0.3^{a}$ & $7.8 \pm 0.3^{a}$ & $7.6 \pm 0.3^{a}$ & $7.4 \pm 0.3^{a}$ & $7.2 \pm 0.3^{\mathrm{a}}$ & $7.1 \pm 0.5^{\mathrm{a}}$ & $7.0 \pm 0.5^{\mathrm{a}}$ & $7.0 \pm 0.3^{a}$ & $6.6 \pm 0.3^{a}$ & $6.4 \pm 0.3^{a}$ \\
\hline & & $6.6 \pm 0.3^{b}$ & $6.5 \pm 0.3^{b}$ & $7.0 \pm 0.3^{b}$ & $6.8 \pm 0.3^{b}$ & $6.5 \pm 0.3^{b}$ & $6.3 \pm 0.3^{b}$ & $6.7 \pm 0.3^{a}$ & $6.5 \pm 0.3^{a}$ & $6.8 \pm 0.3^{\mathrm{a}}$ & $6.9 \pm 0.4^{\mathrm{a}}$ & $6.5 \pm 0.3^{\mathrm{a}}$ & $6.1 \pm 0.3^{a}$ \\
\hline \multirow{2}{*}{$\begin{array}{c}\text { Sage } \\
\text { (Salvia } \\
\text { officinalis L.) }\end{array}$} & \multirow{2}{*}{$\begin{array}{l}\text { after fer- } \\
\text { mentation } \\
\text { after } \\
\text { digestion }\end{array}$} & $7.8 \pm 0.3^{\mathrm{a}}$ & $8.0 \pm 0.3^{a}$ & $7.7 \pm 0.4^{\mathrm{a}}$ & $7.9 \pm 0.2^{\mathrm{a}}$ & $7.7 \pm 0.3^{\mathrm{a}}$ & $7.3 \pm 0.4^{\mathrm{a}, \mathrm{b}}$ & $7.2 \pm 0.3^{\mathrm{a}}$ & $6.7 \pm 0.3^{\mathrm{a}, \mathrm{b}}$ & $6.4 \pm 0.3^{b}$ & $6.4 \pm 0.3^{b}$ & $6.4 \pm 0.3^{b}$ & $6.5 \pm 0.3^{a, b}$ \\
\hline & & $6.9 \pm 0.3^{b}$ & $7.0 \pm 0.2^{b}$ & $6.3 \pm 0.3^{b, c}$ & $6.8 \pm 0.3^{b, c}$ & $6.6 \pm 0.3^{b, c}$ & $6.2 \pm 0.3^{c}$ & $6.8 \pm 0.3^{a, b}$ & $6.3 \pm 0.4^{\mathrm{b}}$ & $6.2 \pm 0.4^{b}$ & $6.2 \pm 0.4^{b}$ & $6.1 \pm 0.3^{b}$ & $6.1 \pm 0.3^{b}$ \\
\hline \multirow{2}{*}{$\begin{array}{c}\text { Chamomile } \\
\text { (Matricaria } \\
\text { chamomilla L.) }\end{array}$} & \multirow{2}{*}{$\begin{array}{l}\text { after fer- } \\
\text { mentation } \\
\text { after } \\
\text { digestion }\end{array}$} & $7.9 \pm 0.3^{\mathrm{a}}$ & $7.9 \pm 0.3^{a}$ & $8.0 \pm 0.3^{\mathrm{a}}$ & $7.9 \pm 0.3^{\mathrm{a}}$ & $7.7 \pm 0.3^{\mathrm{a}}$ & $7.5 \pm 0.3^{a}$ & $7.2 \pm 0.4^{\mathrm{a}}$ & $7.0 \pm 0.5^{\mathrm{a}}$ & $6.8 \pm 0.5^{\mathrm{a}}$ & $6.8 \pm 0.3^{a}$ & $6.5 \pm 0.3^{\mathrm{a}}$ & $6.4 \pm 0.3^{\mathrm{a}}$ \\
\hline & & $6.8 \pm 0.3^{b}$ & $6.3 \pm 0.3^{b}$ & $6.9 \pm 0.3^{b}$ & $6.9 \pm 0.3^{b}$ & $6.5 \pm 0.3^{b}$ & $6.3 \pm 0.3^{b}$ & $6.6 \pm 0.4^{\mathrm{a}}$ & $6.3 \pm 0.4^{\mathrm{a}}$ & $6.6 \pm 0.4^{a}$ & $6.7 \pm 0.4^{\mathrm{a}}$ & $6.5 \pm 0.5^{\mathrm{a}}$ & $6.2 \pm 0.4^{\mathrm{a}}$ \\
\hline \multirow{2}{*}{$\begin{array}{l}\text { Cistus } \\
\text { (Cistus L.) }\end{array}$} & \multirow{2}{*}{$\begin{array}{l}\text { after fer- } \\
\text { mentation } \\
\text { after } \\
\text { digestion }\end{array}$} & $8.1 \pm 0.3^{a}$ & $8.0 \pm 0.3^{a}$ & $7.9 \pm 0.3^{a}$ & $8.0 \pm 0.3^{a}$ & $7.8 \pm 0.3^{\mathrm{a}, \mathrm{b}}$ & $7.4 \pm 0.4^{\mathrm{a}, \mathrm{b}}$ & $7.2 \pm 0.5^{\mathrm{a}}$ & $7.2 \pm 0.3^{a}$ & $7.2 \pm 0.3^{a}$ & $7.6 \pm 0.3^{a}$ & $7.2 \pm 0.3^{a}$ & $6.8 \pm 0.3^{a}$ \\
\hline & & $7.9 \pm 0.4^{\mathrm{a}}$ & $7.8 \pm 0.4^{\mathrm{a}, \mathrm{b}}$ & $7.8 \pm 0.4^{\mathrm{a}, \mathrm{b}}$ & $7.5 \pm 0.3^{a, b}$ & $7.6 \pm 0.3^{a, b}$ & $7.1 \pm 0.2^{b}$ & $6.7 \pm 0.4^{\mathrm{a}}$ & $7.0 \pm 0.4^{\mathrm{a}}$ & $7.0 \pm 0.4^{\mathrm{a}}$ & $7.5 \pm 0.4^{\mathrm{a}}$ & $7.0 \pm 0.4^{\mathrm{a}}$ & $6.6 \pm 0.4^{a}$ \\
\hline \multirow{2}{*}{$\begin{array}{l}\text { Linden } \\
\text { blossom } \\
\text { (Tilia L.) }\end{array}$} & \multirow{2}{*}{$\begin{array}{l}\text { after fer- } \\
\text { mentation } \\
\text { after } \\
\text { digestion }\end{array}$} & $8.2 \pm 0.3^{a}$ & $8.3 \pm 0.3^{a}$ & $8.3 \pm 0.3^{a}$ & $8.2 \pm 0.3^{\mathrm{a}}$ & $7.9 \pm 0.5^{a}$ & $7.8 \pm 0.5^{\mathrm{a}}$ & $7.5 \pm 0.4^{\mathrm{a}}$ & $7.7 \pm 0.3^{a}$ & $7.2 \pm 0.3^{a}$ & $7.5 \pm 0.3^{a}$ & $7.3 \pm 0.3^{\mathrm{a}}$ & $6.9 \pm 0.3^{\mathrm{a}, \mathrm{b}}$ \\
\hline & & $6.9 \pm 0.3^{b}$ & $6.8 \pm 0.3^{\mathrm{b}}$ & $6.9 \pm 0.3^{b}$ & $6.8 \pm 0.3^{\mathrm{b}}$ & $6.6 \pm 0.3^{b}$ & $6.4 \pm 0.3^{b}$ & $7.0 \pm 0.3^{\mathrm{a}}$ & $6.8 \pm 0.3^{\mathrm{a}, \mathrm{b}}$ & $7.0 \pm 0.3^{\mathrm{a}}$ & $6.7 \pm 0.3^{\mathrm{a}, \mathrm{b}}$ & $6.7 \pm 0.3^{\mathrm{a}, \mathrm{b}}$ & $6.3 \pm 0.3^{b}$ \\
\hline \multirow{2}{*}{$\begin{array}{c}\text { Ribwort } \\
\text { plantain } \\
\text { (Plantago } \\
\text { lanceolata L.) }\end{array}$} & \multirow{2}{*}{$\begin{array}{l}\text { after fer- } \\
\text { mentation } \\
\text { after } \\
\text { digestion }\end{array}$} & $8.1 \pm 0.3^{a}$ & $8.1 \pm 0.4^{\mathrm{a}}$ & $8.3 \pm 0.3^{a}$ & $8.3 \pm 0.3^{a}$ & $7.9 \pm 0.2^{\mathrm{a}}$ & $7.5 \pm 0.5^{\mathrm{a}, \mathrm{b}}$ & $7.3 \pm 0.5^{\mathrm{a}}$ & $7.1 \pm 0.3^{\mathrm{a}}$ & $7.1 \pm 0.3^{a}$ & $7.3 \pm 0.3^{a}$ & $7.2 \pm 0.3^{a}$ & $6.8 \pm 0.3^{a}$ \\
\hline & & $7.1 \pm 0.3^{b}$ & $6.7 \pm 0.3^{b, c}$ & $6.6 \pm 0.3^{b, c}$ & $6.5 \pm 0.3^{b, c}$ & $6.5 \pm 0.3^{b, c}$ & $6.2 \pm 0.3^{c}$ & $7.0 \pm 0.4^{\mathrm{a}}$ & $6.7 \pm 0.3^{a}$ & $7.0 \pm 0.4^{\mathrm{a}}$ & $7.2 \pm 0.3^{a}$ & $6.8 \pm 0.3^{a}$ & $6.5 \pm 0.3^{a}$ \\
\hline \multirow[t]{2}{*}{$\begin{array}{l}\text { Marshmallow } \\
\text { (Althaea L.) }\end{array}$} & \multirow{2}{*}{$\begin{array}{l}\text { after fer- } \\
\text { mentation } \\
\text { after } \\
\text { digestion }\end{array}$} & $8.2 \pm 0.3^{a}$ & $8.1 \pm 0.3^{a}$ & $8.0 \pm 0.3^{a}$ & $8.0 \pm 0.3^{a}$ & $7.8 \pm 0.3^{a}$ & $7.4 \pm 0.2^{\mathrm{a}}$ & $7.7 \pm 0.5^{\mathrm{a}}$ & $7.4 \pm 0.3^{a}$ & $7.5 \pm 0.4^{\mathrm{a}}$ & $7.8 \pm 0.3^{a}$ & $7.4 \pm 0.3^{a}$ & $7.0 \pm 0.3^{\mathrm{a}, \mathrm{b}}$ \\
\hline & & $7.2 \pm 0.3^{a, b}$ & $7.2 \pm 0.3^{a, b}$ & $7.2 \pm 0.3^{a, b}$ & $7.3 \pm 0.3^{a}$ & $7.1 \pm 0.3^{a, b}$ & $6.8 \pm 0.3^{b}$ & $7.2 \pm 0.3^{a}$ & $7.1 \pm 0.3^{\mathrm{a}, \mathrm{b}}$ & $7.1 \pm 0.3^{a, b}$ & $7.3 \pm 0.3^{a}$ & $7.0 \pm 0.3^{a, b}$ & $6.6 \pm 0.3^{b}$ \\
\hline
\end{tabular}

$a, b, c$ the same letter indices within a given bacterial species and for a given plant extract mean no statistically significant differences at the significance level of 0.05 . 


\subsection{TPC and Antioxidant Capacities of Plant Extracts}

The phenolic content determined in the tested plant extracts is presented in Table 2. A significant difference was observed in the phenolic content between cistus and linden blossom extracts and the rest of the tested plant extracts. As we mentioned in the materials and methods section marshmallow and plantain extracts were in liquid form while the rest were in powder form. As can be seen from the data presented in Table 2, liquid marshmallow and plantain extracts were not preparations with the lowest total phenolic content, as well as antioxidant capacity. Statistical analysis showed significant differences in the phenolic content, which resulted in the identification of seven homogenous groups (at a $5 \%$ significance level).

Table 2. The TPC and Trolox equivalent antioxidant capacity of plant extracts determined by DPPH, ABTS, and FRAP assays (average values and standard deviations of three replicates).

\begin{tabular}{|c|c|c|c|c|}
\hline \multirow{2}{*}{ Plant Extracts } & \multirow{2}{*}{$\begin{array}{l}\text { Total Phenolic Content } \\
\text { [mg GAE/g of Extract] }\end{array}$} & \multicolumn{3}{|c|}{$\begin{array}{l}\text { Antioxidant Capacities } \\
\text { [mmol TE/g Extract] }\end{array}$} \\
\hline & & DPPH & ABTS & FRAP \\
\hline Valerian (Valeriana officinalis L.) & $30.97 \pm 0.49^{\mathrm{f}}$ & $0.104 \pm 0.003^{b}$ & $0.159 \pm 0.003^{b}$ & $0.090 \pm 0.004^{\mathrm{a}}$ \\
\hline Sage (Salvia officinalis L.) & $61.42 \pm 0.43^{c}$ & $0.137 \pm 0.002^{c}$ & $0.186 \pm 0.004^{c}$ & $0.122 \pm 0.004^{b, c}$ \\
\hline $\begin{array}{c}\text { Chamomile (Matricaria } \\
\text { chamomilla L.) }\end{array}$ & $32.56 \pm 0.24^{\mathrm{e}}$ & $0.099 \pm 0.003^{b}$ & $0.120 \pm 0.003^{\mathrm{a}}$ & $0.092 \pm 0.004^{\mathrm{a}}$ \\
\hline Cistus (Cistus L.) & $106.38 \pm 0.01^{a}$ & $0.154 \pm 0.005^{d}$ & $0.208 \pm 0.004^{d}$ & $0.136 \pm 0.003^{d}$ \\
\hline Linden blossom (Tilia L.) & $104.72 \pm 0.39^{b}$ & $0.161 \pm 0.007^{d}$ & $0.210 \pm 0.007^{\mathrm{d}}$ & $0.133 \pm 0.004^{\mathrm{c}, \mathrm{d}}$ \\
\hline $\begin{array}{c}\text { Ribwort plantain } \\
\text { (Plantago lanceolata L.) }\end{array}$ & $41.84 \pm 0.20^{\mathrm{d}}$ & $0.129 \pm 0.004^{c}$ & $0.150 \pm 0.003^{b}$ & $0.113 \pm 0.006^{b}$ \\
\hline Marshmallow (Althaea L.) & $24.06 \pm 0.34 \mathrm{~g}$ & $0.084 \pm 0.003^{\mathrm{a}}$ & $0.123 \pm 0.005^{\mathrm{a}}$ & $0.098 \pm 0.007^{\mathrm{a}}$ \\
\hline
\end{tabular}

Different letters ${ }^{\left({ }^{a-g}\right)}$ within the same column indicate significant difference at the significance level of 0.05 .

The plant extracts were also screened for DPPH, ABTS, and FRAP radical scavenging activities. It was observed that cistus and linden blossom extracts exhibited the highest DPPH, ABTS, and FRAP radical scavenging activity, whereas the lowest DPPH, ABTS, and FRAP activity was exhibited by marshmallow, chamomile, and valerian extract, respectively. Correlation analysis between TPC and DPPH radical scavenging ability, TPC and ABTS radical scavenging ability, and TPC and FRAP radical scavenging ability showed a high degree of correlation $(\mathrm{r} 2=0.930,0.923$, and 0.931 , respectively). However, the $\mathrm{r} 2$ values of $0.931,0.936$, and 0.883 determined for correlation between DPPH and ABTS radical scavenging activity, DPPH and FRAP radical scavenging activity, and ABTS and FRAP radical scavenging activity, respectively, indicated that mainly phenolic compounds contributed to the total antioxidant activity in the tested plant extracts.

\subsection{Qualitative Analysis of Plant Extracts}

The results of ultrahigh-performance LC (UHPLC)-QTOF-CAD analyses of plant extracts are shown in Tables 3-9. The identified compounds are presented according to their elution order. Compounds were tentatively identified based on their HRMS and HRMS2 spectra. The fragmentation patterns and molecular formulas of the compounds were compared with available literature data. 

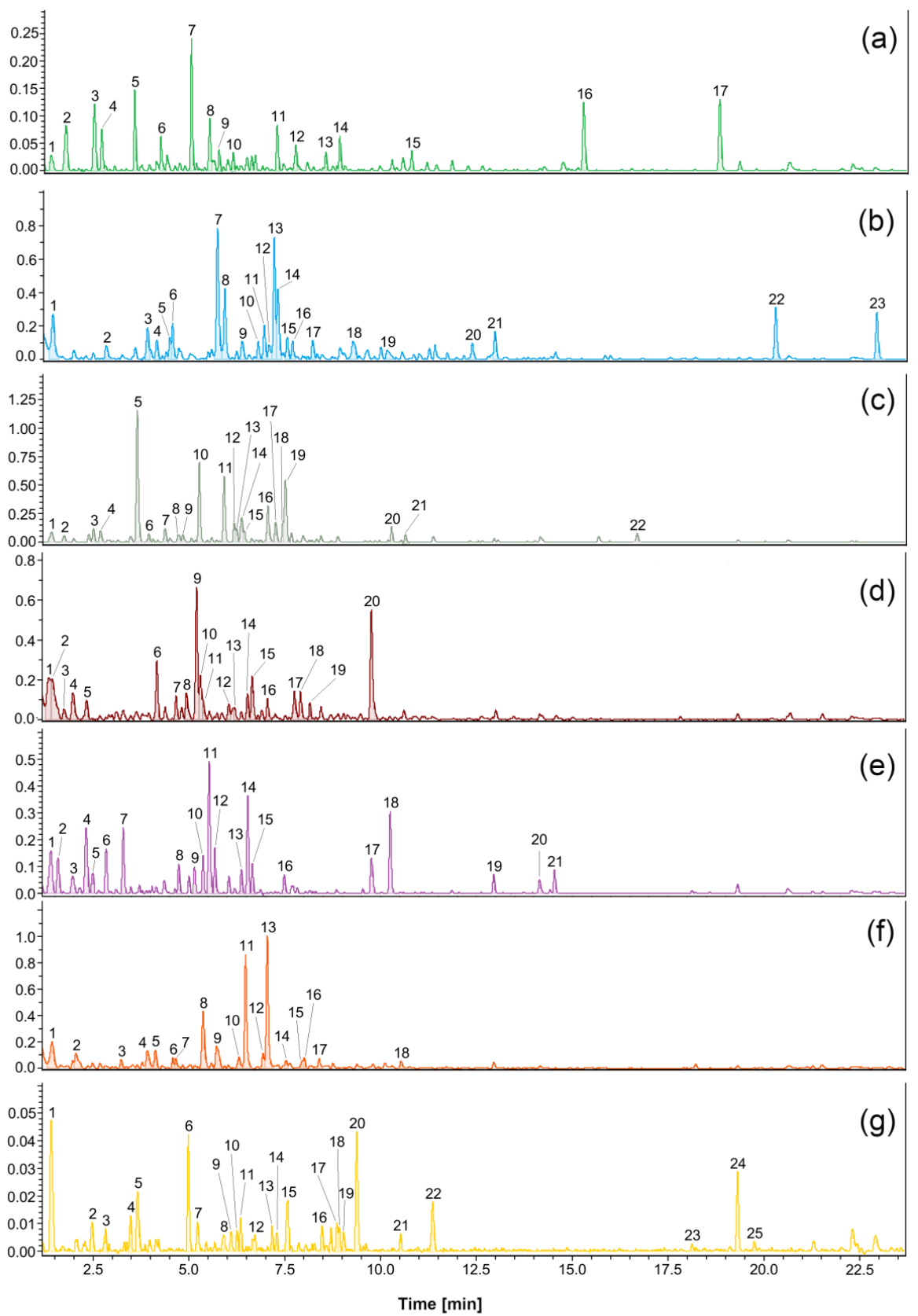

Figure 2. UHPLC-QTOF-CAD chromatograms of the studied plant extracts: (a) valerian (Valeriana officinalis L.); (b) sage (Salvia officinalis L.); (c) chamomile (Matricaria chamomilla L.); (d) cistus (Cistus L.); (e) linden blossom (Tilia L.); (f) ribwort plantain (Plantago lanceolata L.); and (g) marshmallow (Althaea L.). 
Table 3. Compounds identified in the valerian (Valeriana officinalis L.) extract using UHPLC-QTOF-MS/MS. Compound numbers correspond to those indicated in Figure 2.

\begin{tabular}{|c|c|c|c|c|c|c|c|c|}
\hline Peak & $\begin{array}{c}\mathrm{RT} \\
{[\mathrm{min}]}\end{array}$ & $\begin{array}{l}\text { Molecular Ion } \\
\text { [M-H }^{-}\end{array}$ & $\begin{array}{l}\text { Ion } \\
\text { Mode }\end{array}$ & $\begin{array}{c}\text { MS/MS } \\
\text { Fragments }\end{array}$ & Formula & $\begin{array}{c}\text { Error } \\
{[\mathrm{ppm}]}\end{array}$ & mSigma & Tentative Identification \\
\hline 1 & 1.4 & $\begin{array}{l}255.0510 \\
449.1298\end{array}$ & $\begin{array}{l}- \\
-\end{array}$ & $\begin{array}{l}255(100), 193(10) \\
221(100)\end{array}$ & $\begin{array}{l}\mathrm{C}_{11} \mathrm{H}_{11} \mathrm{O}_{7} \\
\mathrm{C}_{18} \mathrm{H}_{25} \mathrm{O}_{13}\end{array}$ & $\begin{array}{l}0.2 \\
0.6\end{array}$ & $\begin{array}{l}13.2 \\
43.8\end{array}$ & $\begin{array}{l}\text { piscidic acid } \\
\text { unidentified }\end{array}$ \\
\hline 2 & 1.8 & 361.1504 & - & $361(100), 199(5)$ & $\mathrm{C}_{16} \mathrm{H}_{25} \mathrm{O}_{9}$ & -0.1 & 11.3 & unidentified \\
\hline 3 & 2.5 & 351.0717 & - & 191(100), 215(11) & $\mathrm{C}_{16} \mathrm{H}_{15} \mathrm{O}_{9}$ & 1.3 & 20.1 & $\begin{array}{l}\text { caffeoylquinic acid } \\
\text { (quinone form) }\end{array}$ \\
\hline 4 & 2.7 & 353.0873 & - & $\begin{array}{c}\text { 173(100), 191(64), } \\
179(45)\end{array}$ & $\mathrm{C}_{16} \mathrm{H}_{17} \mathrm{O}_{9}$ & 1.4 & 11.0 & neochlorogenic acid \\
\hline 5 & 3.5 & 553.1929 & - & 391(100), 195(21) & $\mathrm{C}_{26} \mathrm{H}_{33} \mathrm{O}_{13}$ & -0.4 & 27.4 & $\begin{array}{l}\text { unidentified hexoside } \\
\text { (lignan) }\end{array}$ \\
\hline 6 & 4.2 & $\begin{array}{l}697.2346 \\
553.1916\end{array}$ & $\begin{array}{l}- \\
-\end{array}$ & $\begin{array}{c}373(100), 535(14), \\
181(11) \\
391(100), 195(62)\end{array}$ & $\begin{array}{l}\mathrm{C}_{32} \mathrm{H}_{41} \mathrm{O}_{17} \\
\mathrm{C}_{26} \mathrm{H}_{33} \mathrm{O}_{13}\end{array}$ & $\begin{array}{l}0.4 \\
1.9\end{array}$ & $\begin{array}{c}15.4 \\
118.8\end{array}$ & $\begin{array}{l}\text { hydroxypinoresinol } \\
\text { di-hexoside } \\
\text { unidentified hexoside } \\
\text { (lignan) }\end{array}$ \\
\hline 7 & 5.0 & 681.2390 & - & $357(100)$ & $\mathrm{C}_{32} \mathrm{H}_{41} \mathrm{O}_{16}$ & 1.5 & 2.6 & pinoresinol di-hexoside \\
\hline 8 & 5.5 & 637.2348 & - & $\begin{array}{l}\text { 197(100), 221(52), } \\
341(31)\end{array}$ & $\mathrm{C}_{27} \mathrm{H}_{41} \mathrm{O}_{17}$ & 0.2 & 10.4 & kanokoside $\mathrm{C}$ isomer \\
\hline 9 & 5.7 & 535.1814 & - & 373(100), 181(45) & $\mathrm{C}_{26} \mathrm{H}_{31} \mathrm{O}_{12}$ & 1.4 & 4.8 & $\begin{array}{l}\text { hydroxypinoresinol } \\
\text { hexoside }\end{array}$ \\
\hline 10 & 6.1 & 493.2285 & - & 493(100), 331(47) & $\mathrm{C}_{22} \mathrm{H}_{37} \mathrm{O}_{12}$ & 1.1 & 16.2 & rhodioloside isomer \\
\hline 11 & 7.3 & 519.1869 & - & 357(100), 151(8) & $\mathrm{C}_{26} \mathrm{H}_{31} \mathrm{O}_{11}$ & 0.6 & 7.1 & pinoresinol hexoside \\
\hline 12 & 7.8 & $\begin{array}{l}609.1822 \\
457.1709\end{array}$ & $\begin{array}{l}- \\
-\end{array}$ & $\begin{array}{l}301(100) \\
293(100)\end{array}$ & $\begin{array}{l}\mathrm{C}_{28} \mathrm{H}_{33} \mathrm{O}_{15} \\
\mathrm{C}_{21} \mathrm{H}_{29} \mathrm{O}_{11}\end{array}$ & $\begin{array}{l}0.5 \\
1.4\end{array}$ & $\begin{array}{l}22.0 \\
76.6\end{array}$ & $\begin{array}{l}\text { hesperidin isomer } \\
\text { unidentified }\end{array}$ \\
\hline 13 & 8.5 & $\begin{array}{l}345.1552 \\
347.1708\end{array}$ & $\begin{array}{l}- \\
-\end{array}$ & $\begin{array}{l}345(100) \\
347(100)\end{array}$ & $\begin{array}{l}\mathrm{C}_{16} \mathrm{H}_{25} \mathrm{O}_{8} \\
\mathrm{C}_{16} \mathrm{H}_{27} \mathrm{O}_{8}\end{array}$ & $\begin{array}{l}0.7 \\
1.1\end{array}$ & $\begin{array}{c}4.4 \\
30.1\end{array}$ & $\begin{array}{l}\text { uidentified monoterpene } \\
\text { hexoside (iridoid) } \\
\text { uidentified monoterpene } \\
\text { hexoside (iridoid) }\end{array}$ \\
\hline 14 & 8.9 & 477.2339 & - & 477(100), 315(35) & $\mathrm{C}_{22} \mathrm{H}_{37} \mathrm{O}_{11}$ & 0.5 & 6.4 & $\begin{array}{c}\text { unidentified } \\
\text { monoterpene diglycoside }\end{array}$ \\
\hline 15 & 10.8 & 573.2553 & - & 573(100), 231(4) & $\mathrm{C}_{27} \mathrm{H}_{41} \mathrm{O}_{13}$ & -0.1 & 2.3 & unidentified \\
\hline 16 & 15.3 & 249.1488 & - & 249(100), 163(4) & $\mathrm{C}_{15} \mathrm{H}_{21} \mathrm{O}_{3}$ & 3.4 & 0.7 & valerenolic acid \\
\hline 17 & 18.8 & 291.1592 & - & 291(100), 249(19) & $\mathrm{C}_{17} \mathrm{H}_{23} \mathrm{O}_{4}$ & 3.4 & 3.1 & acetylvalerenolic acid \\
\hline
\end{tabular}

Table 4. Compounds identified in the sage (Salvia officinalis L.) extract using UHPLC-QTOF-MS/MS. Compound numbers correspond to those indicated in Figure 2.

\begin{tabular}{|c|c|c|c|c|c|c|c|c|}
\hline Peak & $\begin{array}{c}\mathrm{RT} \\
{[\mathrm{min}]}\end{array}$ & $\begin{array}{l}\text { Molecular Ion } \\
\text { [M-H }^{-}\end{array}$ & $\begin{array}{l}\text { Ion } \\
\text { Mode }\end{array}$ & $\begin{array}{c}\text { MS/MS } \\
\text { Fragments }\end{array}$ & Formula & $\begin{array}{l}\text { Error } \\
\text { [ppm] }\end{array}$ & mSigma & Tentative Identification \\
\hline 1 & 1.4 & 197.0458 & - & $\begin{array}{c}\text { 179(53), 135(24), } \\
123(23), 195(6), \\
151(4)\end{array}$ & $\mathrm{C}_{9} \mathrm{H}_{9} \mathrm{O}_{5}$ & -1.3 & 10.2 & danshensu isomer \\
\hline 2 & 2.8 & 325.0933 & - & 179(100), 135(14) & $\mathrm{C}_{15} \mathrm{H}_{17} \mathrm{O}_{8}$ & -1.9 & 11.8 & $\begin{array}{c}\text { caffeic } \\
\text { acid-deoxyhexoside }\end{array}$ \\
\hline 3 & 3.9 & 389.1789 & + & $\begin{array}{l}\text { 227(100), 209(98), } \\
\text { 191(56), 131(12) }\end{array}$ & $\mathrm{C}_{18} \mathrm{H}_{29} \mathrm{O}_{9}$ & 4.3 & 13.8 & unidentified \\
\hline 4 & 4.1 & $\begin{array}{l}355.1041 \\
583.2047\end{array}$ & $\begin{array}{l}- \\
-\end{array}$ & $\begin{array}{c}295(100), 265(51), \\
160(17), 193(16), \\
175(10) \\
373(100), 361(98), \\
298(58), 295(33), \\
313(27)\end{array}$ & $\begin{array}{l}\mathrm{C}_{16} \mathrm{H}_{19} \mathrm{O}_{9} \\
\mathrm{C}_{27} \mathrm{H}_{35} \mathrm{O}_{14}\end{array}$ & $\begin{array}{l}-1.9 \\
-2.5\end{array}$ & $\begin{array}{l}19.8 \\
12.4\end{array}$ & $\begin{array}{l}\text { ferulic acid-hexoside } \\
\text { unidentified }\end{array}$ \\
\hline 5 & 4.5 & 493.0628 & - & $\begin{array}{c}299(100), 271(50) \\
241(9)\end{array}$ & $\mathrm{C}_{21} \mathrm{H}_{17} \mathrm{O}_{14}$ & -0.9 & 12.2 & unidentified \\
\hline
\end{tabular}


Table 4. Cont.

\begin{tabular}{|c|c|c|c|c|c|c|c|c|}
\hline Peak & $\begin{array}{c}\mathrm{RT} \\
{[\mathrm{min}]}\end{array}$ & $\begin{array}{l}\text { Molecular Ion } \\
\text { [M-H }^{-}\end{array}$ & $\begin{array}{l}\text { Ion } \\
\text { Mode }\end{array}$ & $\begin{array}{c}\text { MS/MS } \\
\text { Fragments }\end{array}$ & Formula & $\begin{array}{l}\text { Error } \\
\text { [ppm] }\end{array}$ & mSigma & Tentative Identification \\
\hline 6 & 4.6 & 227.1275 & + & $\begin{array}{c}\text { 209(100), 191(87), } \\
\text { 149(55), 167(43), } \\
131(41)\end{array}$ & $\mathrm{C}_{12} \mathrm{H}_{19} \mathrm{O}_{4}$ & 1.4 & 0.6 & unidentified \\
\hline 7 & 5.7 & 461.0731 & - & $285(100), 255(33)$ & $\mathrm{C}_{21} \mathrm{H}_{17} \mathrm{O}_{12}$ & -1.2 & 1.5 & luteoline-oxyhexoside \\
\hline 8 & 5.9 & $\begin{array}{l}593.1522 \\
447.0938\end{array}$ & - & $\begin{array}{c}285(100), 339(12) \\
255(5) \\
284(100), 256(7)\end{array}$ & $\begin{array}{l}\mathrm{C}_{27} \mathrm{H}_{29} \mathrm{O}_{15} \\
\mathrm{C}_{21} \mathrm{H}_{19} \mathrm{O}_{11}\end{array}$ & $\begin{array}{l}-1.7 \\
-1.1\end{array}$ & $\begin{array}{l}6.5 \\
2.4\end{array}$ & $\begin{array}{l}\text { luteoline-hexoside- } \\
\text { deoxyhexoside } \\
\text { luteoline-hexoside }\end{array}$ \\
\hline 9 & 6.4 & 551.1770 & - & $\begin{array}{l}\text { 235(100), 533(88), } \\
295(69), 160(64)\end{array}$ & $\mathrm{C}_{26} \mathrm{H}_{31} \mathrm{O}_{13}$ & 0.1 & 20.5 & unidentified \\
\hline 10 & 6.8 & 577.1200 & - & $269(100)$ & $\mathrm{C}_{26} \mathrm{H}_{25} \mathrm{O}_{15}$ & -0.2 & 11.6 & $\begin{array}{l}\text { apigenin-hexoside- } \\
\text { deoxyhexoside }\end{array}$ \\
\hline 11 & 7.0 & 445.0779 & - & $269(100)$ & $\mathrm{C}_{21} \mathrm{H}_{17} \mathrm{O}_{11}$ & -0.6 & 9.0 & apigenin-oxyhexoside \\
\hline 12 & 7.1 & 431.0984 & - & $268(100)$ & $\mathrm{C}_{21} \mathrm{H}_{19} \mathrm{O}_{10}$ & 0.0 & 3.3 & apigenin-hexoside \\
\hline 13 & 7.2 & 359.0768 & - & $\begin{array}{c}\text { 161(100), 197(77), } \\
179(32)\end{array}$ & $\mathrm{C}_{18} \mathrm{H}_{15 \mathrm{O}_{8}}$ & 1.1 & 4.3 & rosmarinic acid \\
\hline 14 & 7.3 & 555.1141 & & $\begin{array}{l}\text { 359(100), 161(22), } \\
135(16), 193(14)\end{array}$ & $\mathrm{C}_{27} \mathrm{H}_{23} \mathrm{O}_{13}$ & 0.7 & 7.9 & salvianolic acid $\mathrm{K}$ isomer \\
\hline 15 & 7.5 & 475.0881 & - & 284(100), 299(61) & $\mathrm{C}_{22} \mathrm{H}_{19} \mathrm{O}_{12}$ & 0.2 & 24.7 & $\begin{array}{l}\text { hispiludin/diosmetin- } \\
\text { oxyhexoside }\end{array}$ \\
\hline 16 & 7.7 & $\begin{array}{l}463.1224 \\
609.1771\end{array}$ & $\begin{array}{l}+ \\
+\end{array}$ & $\begin{array}{c}301(100) \\
301(100), 463(4)\end{array}$ & $\begin{array}{l}\mathrm{C}_{22} \mathrm{H}_{23} \mathrm{O}_{11} \\
\mathrm{C}_{35} \mathrm{H}_{29} \mathrm{O}_{10}\end{array}$ & $\begin{array}{c}2.3 \\
-2.7\end{array}$ & $\begin{array}{c}8.8 \\
44.0\end{array}$ & $\begin{array}{l}\text { kaempferide-hexoside } \\
\text { kaempferide-hexoside- } \\
\text { deoxyhexoside }\end{array}$ \\
\hline 17 & 8.2 & 769.1637 & - & $\begin{array}{c}285(100), 255(26) \\
575(4)\end{array}$ & $\mathrm{C}_{36} \mathrm{H}_{33} \mathrm{O}_{19}$ & -2.1 & 22.5 & $\begin{array}{l}\text { luteolin-oxyhexoside- } \\
\text { pentoside-ferulic } \\
\text { acid }\end{array}$ \\
\hline 18 & 9.3 & $\begin{array}{l}753.1679 \\
621.1842\end{array}$ & - & $\begin{array}{c}\text { 269(100), 486(7), } \\
193(4) \\
313(100), 297(44)\end{array}$ & $\begin{array}{l}\mathrm{C}_{36} \mathrm{H}_{33} \mathrm{O}_{18} \\
\mathrm{C}_{29} \mathrm{H}_{33} \mathrm{O}_{15}\end{array}$ & $\begin{array}{l}-0.9 \\
-2.7\end{array}$ & $\begin{array}{l}18.8 \\
14.2\end{array}$ & $\begin{array}{l}\text { apigenin-oxyhexoside- } \\
\text { pentoside-ferulic acid } \\
\text { unidentified }\end{array}$ \\
\hline 19 & 10.2 & 711.3968 & - & $503(100), 453(10)$ & $\mathrm{C}_{37} \mathrm{H}_{59} \mathrm{O}_{13}$ & -1.0 & 19.4 & unidentified \\
\hline 20 & 12.4 & 493.1140 & - & $\begin{array}{c}\text { 359(100), 323(40), } \\
\text { 135(21), 179(16), } \\
295(14)\end{array}$ & $\mathrm{C}_{26} \mathrm{H}_{21} \mathrm{O}_{10}$ & 0.0 & 13.0 & unidentified \\
\hline 21 & 13.0 & 327.2178 & - & $\begin{array}{l}\text { 327(100), 211(12), } \\
229(5), 171(3)\end{array}$ & $\mathrm{C}_{18} \mathrm{H}_{31} \mathrm{O}_{5}$ & -0.3 & 6.2 & unidentified \\
\hline 22 & 20.3 & 329.1760 & - & $285(100)$ & $\mathrm{C}_{20} \mathrm{H}_{25} \mathrm{O}_{4}$ & -0.4 & 13.3 & carnosol isomer \\
\hline 23 & 22.9 & 331.1926 & - & $287(100)$ & $\mathrm{C}_{20} \mathrm{H}_{27} \mathrm{O}_{4}$ & -3.3 & 11.9 & carnosoic acid isomer \\
\hline
\end{tabular}

Table 5. Compounds identified in the chamomile (Matricaria chamomilla L.) extract using UHPLC-QTOF-MS/MS. Compound numbers correspond to those indicated in Figure 2.

\begin{tabular}{|c|c|c|c|c|c|c|c|c|}
\hline Peak & $\left.\begin{array}{c}\text { RT } \\
{[\mathrm{min}]}\end{array}\right]$ & $\begin{array}{l}\text { Molecular Ion } \\
{\text { [M-H }]^{-}}^{-}\end{array}$ & $\begin{array}{l}\text { Ion } \\
\text { Mode }\end{array}$ & $\begin{array}{c}\text { MS/MS } \\
\text { Fragments }\end{array}$ & Formula & $\begin{array}{l}\text { Error } \\
\text { [ppm] }\end{array}$ & mSigma & Tentative Identification \\
\hline 1 & 1.4 & $\begin{array}{l}315.0719 \\
329.0874\end{array}$ & - & $\begin{array}{l}315(100), 152(10) \\
167(100), 329(28)\end{array}$ & $\begin{array}{l}\mathrm{C}_{13} \mathrm{H}_{15} \mathrm{O}_{9} \\
\mathrm{C}_{14} \mathrm{H}_{17} \mathrm{O}_{9}\end{array}$ & $\begin{array}{l}0.7 \\
1.2\end{array}$ & $\begin{array}{c}3.8 \\
18.3\end{array}$ & $\begin{array}{l}\text { protocatechuoyl- } \\
\text { hexoside } \\
\text { vanilic acid-hexoside }\end{array}$ \\
\hline 2 & 1.7 & 353.0877 & - & $\begin{array}{c}\text { 191(100), 179(40) } \\
135(30)\end{array}$ & $\mathrm{C}_{16} \mathrm{H}_{17} \mathrm{O}_{9}$ & 0.2 & 1.6 & chlorogenic acid \\
\hline 3 & 2.5 & 353.0875 & - & 191(100) & $\mathrm{C}_{16} \mathrm{H}_{17} \mathrm{O}_{9}$ & 1.0 & 7.7 & caffeoylqunic acid \\
\hline 4 & 2.7 & 353.0874 & - & 191(100), 173(83) & $\mathrm{C}_{16} \mathrm{H}_{17} \mathrm{O}_{9}$ & 1.3 & 4.5 & caffeoylqunic acid \\
\hline 5 & 3.7 & $\begin{array}{l}355.1035 \\
515.1201\end{array}$ & - & $\begin{array}{c}\text { 193(100), 149(44) } \\
353(100), 191(59) \\
179(22), 135(12)\end{array}$ & $\begin{array}{l}\mathrm{C}_{16} \mathrm{H}_{19} \mathrm{O}_{9} \\
\mathrm{C}_{25} \mathrm{H}_{23} \mathrm{O}_{12}\end{array}$ & $\begin{array}{c}0.0 \\
-1.2\end{array}$ & $\begin{array}{c}5.5 \\
34.5\end{array}$ & $\begin{array}{l}\text { ferulic acid hexoside } \\
\text { dicaffeoylqunic acid }\end{array}$ \\
\hline
\end{tabular}


Table 5. Cont.

\begin{tabular}{|c|c|c|c|c|c|c|c|c|}
\hline Peak & $\begin{array}{c}\text { RT } \\
{[\mathrm{min}]}\end{array}$ & $\begin{array}{l}\text { Molecular Ion } \\
{ }_{[\mathrm{M}-\mathrm{H}]^{-}}\end{array}$ & $\begin{array}{l}\text { Ion } \\
\text { Mode }\end{array}$ & $\begin{array}{c}\text { MS/MS } \\
\text { Fragments }\end{array}$ & Formula & $\begin{array}{r}\text { Error } \\
\text { [ppm] }\end{array}$ & mSigma & Tentative Identification \\
\hline 6 & 4.0 & 639.1564 & - & $\begin{array}{c}313(100), 477(53), \\
270(36)\end{array}$ & $\mathrm{C}_{28} \mathrm{H}_{31} \mathrm{O}_{17}$ & 0.4 & 11.4 & $\begin{array}{l}\text { isorhamnetin-di- } \\
\text { hexoside }\end{array}$ \\
\hline 7 & 4.4 & $\begin{array}{l}479.0834 \\
609.1464\end{array}$ & $\begin{array}{l}- \\
-\end{array}$ & $\begin{array}{c}317(100), 287(10), \\
165(6) \\
284(100), 447(38)\end{array}$ & $\begin{array}{l}\mathrm{C}_{21} \mathrm{H}_{19} \mathrm{O}_{13} \\
\mathrm{C}_{27} \mathrm{H}_{29} \mathrm{O}_{16}\end{array}$ & $\begin{array}{l}-0.7 \\
-0.4\end{array}$ & $\begin{array}{l}15.1 \\
28.5\end{array}$ & $\begin{array}{l}\text { myricetin-oxyhexoside } \\
\text { luteolin-di-hexoside }\end{array}$ \\
\hline 8 & 4.7 & $\begin{array}{l}367.1037 \\
463.0883\end{array}$ & $\begin{array}{l}- \\
-\end{array}$ & $\begin{array}{c}367(100), 173(19) \\
193(9), 134(7) \\
300(100), 227(8)\end{array}$ & $\begin{array}{l}\mathrm{C}_{17} \mathrm{H}_{19} \mathrm{O}_{9} \\
\mathrm{C}_{21} \mathrm{H}_{19} \mathrm{O}_{12}\end{array}$ & $\begin{array}{l}-0.6 \\
-0.2\end{array}$ & $\begin{array}{l}6.5 \\
9.7\end{array}$ & $\begin{array}{l}\text { feruloylquinic acid } \\
\text { quercetin- hexoside }\end{array}$ \\
\hline 9 & 4.8 & 609.1469 & - & $301(100)$ & $\mathrm{C}_{27} \mathrm{H}_{29} \mathrm{O}_{16}$ & -1.3 & 5.3 & $\begin{array}{l}\text { quercetin-hexoside- } \\
\text { deoxyhexoside }\end{array}$ \\
\hline 10 & 5.3 & 355.1039 & - & 193(100), 149(27) & $\mathrm{C}_{16} \mathrm{H}_{19} \mathrm{O}_{9}$ & -1.3 & 9.5 & ferulic acid hexoside \\
\hline 11 & 5.9 & $\begin{array}{l}593.1516 \\
447.0937\end{array}$ & $\begin{array}{l}- \\
-\end{array}$ & $\begin{array}{l}285(100) \\
284(100)\end{array}$ & $\begin{array}{l}\mathrm{C}_{27} \mathrm{H}_{29} \mathrm{O}_{15} \\
\mathrm{C}_{21} \mathrm{H}_{19} \mathrm{O}_{11}\end{array}$ & $\begin{array}{l}-0.6 \\
-1.0\end{array}$ & $\begin{array}{l}5.0 \\
3.7\end{array}$ & $\begin{array}{l}\text { kaempferol-hexoside- } \\
\text { deoxyhexoside } \\
\text { kaempferol-hexoside }\end{array}$ \\
\hline 12 & 6.2 & 493.1002 & - & $\begin{array}{c}\text { 331(100), 287(83), } \\
315(55)\end{array}$ & $\mathrm{C}_{22} \mathrm{H}_{21} \mathrm{O}_{13}$ & -2.9 & 6.4 & petuletin-hexoside \\
\hline 13 & 6.2 & 467.1679 & - & $\begin{array}{c}323(100), 305(66) \\
189(25)\end{array}$ & $\mathrm{C}_{26} \mathrm{H}_{27} \mathrm{O}_{8}$ & 6.8 & 28.6 & unidentified \\
\hline 14 & 6.4 & $\begin{array}{l}623.1624 \\
515.1201\end{array}$ & $\begin{array}{l}- \\
-\end{array}$ & $\begin{array}{c}315(100), 300(88) \\
271(28) \\
353(100), 191(36) \\
179(26), 135(11)\end{array}$ & $\begin{array}{l}\mathrm{C}_{28} \mathrm{H}_{31} \mathrm{O}_{16} \\
\mathrm{C}_{25} \mathrm{H}_{23} \mathrm{O}_{12}\end{array}$ & $\begin{array}{l}-1.1 \\
-1.3\end{array}$ & $\begin{array}{l}29.1 \\
5.4\end{array}$ & $\begin{array}{c}\text { isorhamnetin-hexoside- } \\
\text { deoxyhexoside } \\
\text { dicaffeoylqunic acid }\end{array}$ \\
\hline 15 & 6.5 & $\begin{array}{l}681.1674 \\
515.1202\end{array}$ & - & $\begin{array}{c}313(100), 270(45) \\
519(27), 477(24) \\
353(100), 191(71) \\
179(25)\end{array}$ & $\begin{array}{l}\mathrm{C}_{30} \mathrm{H}_{33} \mathrm{O}_{18} \\
\mathrm{C}_{25} \mathrm{H}_{23} \mathrm{O}_{12}\end{array}$ & $\begin{array}{l}-0.3 \\
-1.3\end{array}$ & $\begin{array}{l}13.3 \\
25.4\end{array}$ & $\begin{array}{c}\text { isorhamnetin-hexoside- } \\
\text { acylhexoside } \\
\text { dicaffeoylqunic acid }\end{array}$ \\
\hline 16 & 7.1 & 431.0991 & - & $268(100)$ & $\mathrm{C}_{21} \mathrm{H}_{19} \mathrm{O}_{10}$ & -1.6 & 7.7 & apigenin-hexoside \\
\hline 17 & 7.3 & $\begin{array}{l}515.1204 \\
445.1727\end{array}$ & $\begin{array}{l}- \\
-\end{array}$ & $\begin{array}{c}353(100), 179(18) \\
191(15), 135(6) \\
445(100), 243(44) \\
183(6), 139(3)\end{array}$ & $\begin{array}{l}\mathrm{C}_{25} \mathrm{H}_{23} \mathrm{O}_{12} \\
\mathrm{C}_{20} \mathrm{H}_{29} \mathrm{O}_{11}\end{array}$ & $\begin{array}{l}-1.7 \\
-2.5\end{array}$ & $\begin{array}{c}7.2 \\
22.4\end{array}$ & $\begin{array}{l}\text { dicaffeoylqunic acid } \\
\text { unidetified }\end{array}$ \\
\hline 18 & 7.5 & $\begin{array}{l}479.1169 \\
609.1773\end{array}$ & $\begin{array}{l}+ \\
+\end{array}$ & $\begin{array}{c}317(100) \\
301(100), 463(4)\end{array}$ & $\begin{array}{l}\mathrm{C}_{29} \mathrm{H}_{19} \mathrm{O}_{7} \\
\mathrm{C}_{35} \mathrm{H}_{29} \mathrm{O}_{10}\end{array}$ & $\begin{array}{l}-9.1 \\
-2.9\end{array}$ & $\begin{array}{l}14.9 \\
41.0\end{array}$ & $\begin{array}{l}\text { isorhamnetin-hexoside } \\
\text { kaempferide-hexoside- } \\
\text { deoxyhexoside }\end{array}$ \\
\hline 19 & 7.5 & $\begin{array}{l}477.1045 \\
711.2156\end{array}$ & $\begin{array}{l}- \\
-\end{array}$ & $\begin{array}{c}477(100), 299(65) \\
271(63), 315(46) \\
711(100), 549(20), \\
433(10), 271(66)\end{array}$ & $\begin{array}{l}\mathrm{C}_{22} \mathrm{H}_{21} \mathrm{O}_{12} \\
\mathrm{C}_{32} \mathrm{H}_{39} \mathrm{O}_{18}\end{array}$ & $\begin{array}{l}-1.4 \\
-2.0\end{array}$ & $\begin{array}{c}10.3 \\
9.1\end{array}$ & $\begin{array}{c}\text { isorhamnetin-hexoside } \\
\text { unidentified-hexoside } \\
\text { deoxyhexoside- } \\
\text { pentoside } \\
\text { (flavonoid) }\end{array}$ \\
\hline 20 & 10.3 & 475.1216 & + & $271(100)$ & $\mathrm{C}_{23} \mathrm{H}_{23} \mathrm{O}_{11}$ & 3.9 & 7.4 & apigenin-acylhexoside \\
\hline 21 & 10.6 & 519.1141 & - & $\begin{array}{l}\text { 271(72), 299(46), } \\
313(16), 151(7)\end{array}$ & $\mathrm{C}_{24} \mathrm{H}_{23} \mathrm{O}_{13}$ & 0.5 & 1.9 & $\begin{array}{l}\text { isorhamnetin- } \\
\text { acylhexoside }\end{array}$ \\
\hline 22 & 16.7 & 447.2009 & + & $\begin{array}{c}\text { 219(100), 181(65), } \\
\text { 231(30), } 411(25), \\
358(23)\end{array}$ & $\mathrm{C}_{24} \mathrm{H}_{31} \mathrm{O}_{8}$ & 1.0 & 8.8 & unidentified \\
\hline
\end{tabular}


Table 6. Compounds identified in the cistus (Cistus L.) extract using UHPLC-QTOF-MS/MS. Compound numbers correspond to those indicated in Figure 2.

\begin{tabular}{|c|c|c|c|c|c|c|c|c|}
\hline Peak & $\begin{array}{c}\text { RT } \\
{[\mathrm{min}]}\end{array}$ & $\begin{array}{l}\text { Molecular Ion } \\
\text { [M-H }^{-} \\
\end{array}$ & $\begin{array}{c}\text { Ion } \\
\text { Mode }\end{array}$ & $\begin{array}{c}\text { MS/MS } \\
\text { Fragments }\end{array}$ & Formula & $\begin{array}{c}\text { Error } \\
{[\mathrm{ppm}]}\end{array}$ & mSigma & Tentative Identification \\
\hline 1 & 1.4 & 305.0667 & - & $\begin{array}{c}\text { 305(100), 219(12), } \\
165(8)\end{array}$ & $\mathrm{C}_{15} \mathrm{H}_{13} \mathrm{O}_{7}$ & -0.1 & 5.1 & gallocatechin isomer \\
\hline 2 & 1.4 & 469.0054 & - & 425(100), 299(24) & $\mathrm{C}_{21} \mathrm{H}_{9} \mathrm{O}_{13}$ & -1.0 & 12.5 & $\begin{array}{l}\text { valoneic acid dilacton } \\
\text { isomer }\end{array}$ \\
\hline 3 & 1.8 & 633.0743 & - & $\begin{array}{l}\text { 301(100), 275(49), } \\
229(23), 257(22)\end{array}$ & $\mathrm{C}_{27} \mathrm{H}_{21} \mathrm{O}_{18}$ & -1.5 & 1.1 & strictinin isomer \\
\hline 4 & 2.0 & $\begin{array}{l}305.0672 \\
591.1370\end{array}$ & $\begin{array}{l}- \\
-\end{array}$ & $\begin{array}{c}\text { 305(100), 219(13), } \\
261(8), 179(8) \\
285(100), 305(12)\end{array}$ & $\begin{array}{l}\mathrm{C}_{15} \mathrm{H}_{13} \mathrm{O}_{7} \\
\mathrm{C}_{27} \mathrm{H}_{27} \mathrm{O}_{15}\end{array}$ & $\begin{array}{l}-1.9 \\
-2.5\end{array}$ & $\begin{array}{c}5.4 \\
41.7\end{array}$ & $\begin{array}{c}\text { gallocatechin isomer } \\
\text { unidentified }\end{array}$ \\
\hline 5 & 2.3 & $\begin{array}{l}289.0722 \\
633.0742\end{array}$ & $\begin{array}{l}- \\
-\end{array}$ & $\begin{array}{c}289(100), 245(17) \\
203(9) \\
301(100), 275(41) \\
257(22), 229(11)\end{array}$ & $\begin{array}{l}\mathrm{C}_{15} \mathrm{H}_{13} \mathrm{O}_{6} \\
\mathrm{C}_{27} \mathrm{H}_{21} \mathrm{O}_{18}\end{array}$ & $\begin{array}{l}-1.6 \\
-1.4\end{array}$ & $\begin{array}{c}1.1 \\
35.6\end{array}$ & $\begin{array}{l}\text { epicatechin } \\
\text { strictinin isomer }\end{array}$ \\
\hline 6 & 4.2 & 479.0846 & - & $316(100), 271(83)$ & $\mathrm{C}_{21} \mathrm{H}_{19} \mathrm{O}_{13}$ & -3.2 & 13.7 & myricetin-hexoside \\
\hline 7 & 4.7 & 327.1458 & - & $327(100), 165(10)$ & $\mathrm{C}_{16} \mathrm{H}_{23} \mathrm{O}_{7}$ & -2.8 & 9.2 & unidentified \\
\hline 8 & 5.0 & 449.0738 & - & $316(100), 271(68)$ & $\mathrm{C}_{20} \mathrm{H}_{17} \mathrm{O}_{12}$ & -2.8 & 4.1 & myricetin-pentoside \\
\hline 9 & 5.2 & 463.0897 & - & $\begin{array}{c}\text { 316(100), 271(91), } \\
179(2)\end{array}$ & $\mathrm{C}_{21} \mathrm{H}_{19} \mathrm{O}_{12}$ & -3.2 & 3.5 & $\begin{array}{c}\text { myricetin- } \\
\text { deoxyhexoside }\end{array}$ \\
\hline 10 & 5.3 & 463.0898 & - & 271(100), 300(83) & $\mathrm{C}_{21} \mathrm{H}_{19} \mathrm{O}_{12}$ & -3.6 & 0.8 & quercetin-hexoside \\
\hline 11 & 5.4 & 609.1474 & - & 271(100), 300(76) & $\mathrm{C}_{27} \mathrm{H}_{29} \mathrm{O}_{16}$ & -2.1 & 6.5 & $\begin{array}{l}\text { quercetin-hexoside- } \\
\text { deoxyhexoside }\end{array}$ \\
\hline 12 & 6.1 & 433.0785 & - & $\begin{array}{l}\text { 300(100), 271(86), } \\
255(35), 243(18)\end{array}$ & $\mathrm{C}_{20} \mathrm{H}_{17} \mathrm{O}_{11}$ & -2.0 & 5.8 & quercetin-pentoside \\
\hline 13 & 6.2 & 449.1075 & + & $\begin{array}{c}\text { 197(100), 287(34) } \\
179(11)\end{array}$ & $\mathrm{C}_{21} \mathrm{H}_{21} \mathrm{O}_{11}$ & 0.9 & 11.9 & unidentified \\
\hline 14 & 6.5 & $\begin{array}{l}577.1573 \\
447.0944\end{array}$ & $\begin{array}{l}- \\
-\end{array}$ & $\begin{array}{c}283(100), 255(57) \\
285(55), 431(24) \\
255(100), 227(87) \\
284(49)\end{array}$ & $\begin{array}{l}\mathrm{C}_{27} \mathrm{H}_{29} \mathrm{O}_{14} \\
\mathrm{C}_{21} \mathrm{H}_{19} \mathrm{O}_{11}\end{array}$ & $\begin{array}{l}-1.8 \\
-2.6\end{array}$ & $\begin{array}{l}15.6 \\
11.4\end{array}$ & $\begin{array}{l}\text { kaempferol-di- } \\
\text { deoxyhexoside } \\
\text { kaempferol-hexoside }\end{array}$ \\
\hline 15 & 6.7 & 773.1905 & + & 147(100), 319(18) & $\mathrm{C}_{36} \mathrm{H}_{37} \mathrm{O}_{19}$ & 2.4 & 44.2 & $\begin{array}{c}\text { myricetin-di- } \\
\text { deoxyhexoside-hexoside }\end{array}$ \\
\hline 16 & 7.1 & 507.2219 & + & 219(100), 189(5) & $\mathrm{C}_{26} \mathrm{H}_{35} \mathrm{O}_{10}$ & 1.1 & 9.2 & unidentified \\
\hline 17 & 7.8 & 523.2199 & - & $\begin{array}{c}\text { 475(100), 327(17) } \\
149(7)\end{array}$ & $\mathrm{C}_{26} \mathrm{H}_{35} \mathrm{O}_{11}$ & -2.7 & 3.3 & unidentified \\
\hline 18 & 7.9 & 627.1315 & + & 147(100), 319(58) & $\mathrm{C}_{30} \mathrm{H}_{27} \mathrm{O}_{15}$ & 4.8 & 37.4 & $\begin{array}{l}\text { myricetin-hexoside- } \\
\text { deoxyhexoside }\end{array}$ \\
\hline 19 & 8.2 & 551.2145 & - & $\begin{array}{c}329(100), 269(36) \\
314(35)\end{array}$ & $\mathrm{C}_{27} \mathrm{H}_{35} \mathrm{O}_{12}$ & -1.9 & 7.2 & unidentified \\
\hline 20 & 9.8 & 595.1432 & + & 147(100), 287(39) & $\mathrm{C}_{30} \mathrm{H}_{27} \mathrm{O}_{13}$ & 2.5 & 13.1 & unidentified \\
\hline
\end{tabular}


Table 7. Compounds identified in the linden blossom (Tilia L.) extract using UHPLC-QTOF-MS/MS. Compound numbers correspond to those indicated in Figure 2.

\begin{tabular}{|c|c|c|c|c|c|c|c|c|}
\hline Peak & $\begin{array}{c}\mathrm{RT} \\
{[\mathrm{min}]}\end{array}$ & $\begin{array}{c}\text { Molecular Ion } \\
\text { [M-H }^{-} \\
\end{array}$ & $\begin{array}{c}\text { Ion } \\
\text { Mode }\end{array}$ & $\begin{array}{c}\text { MS/MS } \\
\text { Fragments }\end{array}$ & Formula & $\begin{array}{c}\text { Error } \\
{[\mathrm{ppm}]}\end{array}$ & mSigma & Tentative Identification \\
\hline 1 & 1.4 & 315.0715 & - & $315(100), 152(8)$ & $\mathrm{C}_{13} \mathrm{H}_{15} \mathrm{O}_{9}$ & 2.0 & 0.3 & $\begin{array}{c}\text { protocatechuoyl- } \\
\text { hexoside }\end{array}$ \\
\hline 2 & 1.6 & 153.0187 & - & 153(100), 109(40) & $\mathrm{C}_{7} \mathrm{H}_{5} \mathrm{O}_{4}$ & 4.3 & 14.4 & protocatechuic acid \\
\hline 3 & 2.0 & 577.1340 & - & $289(100), 407(72)$ & $\mathrm{C}_{30} \mathrm{H}_{25} \mathrm{O}_{12}$ & 2.1 & 4.2 & procyanidin isomer \\
\hline 4 & 2.3 & 289.0712 & - & $289(100)$ & $\mathrm{C}_{15} \mathrm{H}_{13} \mathrm{O}_{6}$ & 1.8 & 2.3 & catechin \\
\hline 5 & 2.5 & $\begin{array}{l}353.0867 \\
577.1341 \\
\end{array}$ & $\begin{array}{l}- \\
- \\
\end{array}$ & $\begin{array}{c}191(100), 353(6) \\
289(100), 407(71)\end{array}$ & $\begin{array}{l}\mathrm{C}_{16} \mathrm{H}_{17} \mathrm{O}_{9} \\
\mathrm{C}_{30} \mathrm{H}_{25} \mathrm{O}_{12} \\
\end{array}$ & $\begin{array}{l}3.1 \\
1.8 \\
\end{array}$ & $\begin{array}{c}23.4 \\
7.4\end{array}$ & $\begin{array}{l}\text { chlorogenic acid } \\
\text { procyanidin isomer }\end{array}$ \\
\hline 6 & 2.9 & 577.1344 & - & $289(100), 407(72)$ & $\mathrm{C}_{30} \mathrm{H}_{25} \mathrm{O}_{12}$ & 1.2 & 13.7 & procyanidin isomer \\
\hline 7 & 3.3 & 289.0714 & - & $\begin{array}{c}\text { 289(100), 245(19), } \\
203(9)\end{array}$ & $\mathrm{C}_{15} \mathrm{H}_{13} \mathrm{O}_{6}$ & 1.3 & 5.2 & catechin \\
\hline 8 & 4.8 & 609.1457 & - & $\begin{array}{c}\text { 299(100), 271(63) } \\
447(12)\end{array}$ & $\mathrm{C}_{27} \mathrm{H}_{29} \mathrm{O}_{16}$ & 0.7 & 17.2 & $\begin{array}{l}\text { quercetin-hexoside- } \\
\text { deoxyhexoside }\end{array}$ \\
\hline 9 & 5.2 & 593.1516 & - & $\begin{array}{c}\text { 283(100), 285(43) } \\
447(36)\end{array}$ & $\mathrm{C}_{27} \mathrm{H}_{29} \mathrm{O}_{15}$ & -0.7 & 23.8 & $\begin{array}{l}\text { kaempferol-hexoside- } \\
\text { deoxyhexoside }\end{array}$ \\
\hline 10 & 5.4 & 609.1469 & - & 271(100), 300(68) & $\mathrm{C}_{27} \mathrm{H}_{29} \mathrm{O}_{16}$ & -1.2 & 10.4 & $\begin{array}{l}\text { quercetin-hexoside- } \\
\text { deoxyhexoside }\end{array}$ \\
\hline 11 & 5.5 & $\begin{array}{l}465.1014 \\
595.1634\end{array}$ & $\begin{array}{l}+ \\
+\end{array}$ & $\begin{array}{l}303(100) \\
287(100)\end{array}$ & $\begin{array}{l}\mathrm{C}_{21} \mathrm{H}_{21} \mathrm{O}_{12} \\
\mathrm{C}_{27} \mathrm{H}_{31} \mathrm{O}_{15}\end{array}$ & $\begin{array}{l}2.8 \\
3.9\end{array}$ & $\begin{array}{l}4.9 \\
7.6\end{array}$ & $\begin{array}{l}\text { quercetin-hexoside } \\
\text { kaempferol-hexoside- } \\
\text { deoxyhexoside }\end{array}$ \\
\hline 12 & 5.7 & $\begin{array}{l}449.1066 \\
595.1634\end{array}$ & $\begin{array}{l}+ \\
+\end{array}$ & $\begin{array}{c}303(100) \\
303(100), 449(4)\end{array}$ & $\begin{array}{l}\mathrm{C}_{21} \mathrm{H}_{21} \mathrm{O}_{11} \\
\mathrm{C}_{27} \mathrm{H}_{31} \mathrm{O}_{15}\end{array}$ & $\begin{array}{l}2.7 \\
2.8\end{array}$ & $\begin{array}{l}3.8 \\
2.4\end{array}$ & $\begin{array}{c}\text { quercetin-deoxyhexoside } \\
\text { quercetin-di- } \\
\text { deoxyhexoside }\end{array}$ \\
\hline 13 & 6.4 & 595.1640 & + & 287(100) & $\mathrm{C}_{27} \mathrm{H}_{31} \mathrm{O}_{15}$ & 3.0 & 5.3 & $\begin{array}{l}\text { kaempferol-hexoside- } \\
\text { deoxyhexoside }\end{array}$ \\
\hline 14 & 6.6 & $\begin{array}{l}449.1062 \\
579.1690 \\
711.2102\end{array}$ & $\begin{array}{l}+ \\
+ \\
+\end{array}$ & $\begin{array}{c}287(100) \\
287(100), 433(4) \\
287(100), 433(4)\end{array}$ & $\begin{array}{l}\mathrm{C}_{21} \mathrm{H}_{21} \mathrm{O}_{11} \\
\mathrm{C}_{27} \mathrm{H}_{31} \mathrm{O}_{14} \\
\mathrm{C}_{32} \mathrm{H}_{39} \mathrm{O}_{18}\end{array}$ & $\begin{array}{l}3.6 \\
3.2 \\
4.1\end{array}$ & $\begin{array}{c}4.9 \\
6.0 \\
11.5\end{array}$ & $\begin{array}{l}\text { kaempferol-hexoside } \\
\text { kaempferol-di- } \\
\text { deoxyhexoside } \\
\text { kaempferol-di- } \\
\text { deoxyhexoside- } \\
\text { pentoside }\end{array}$ \\
\hline 15 & 6.7 & 447.0937 & - & 271(100), 300(73) & $\mathrm{C}_{21} \mathrm{H}_{19} \mathrm{O}_{11}$ & -0.9 & 3.1 & quercetin-deoxyhexoside \\
\hline 16 & 7.5 & 463.0886 & - & $301(100)$ & $\mathrm{C}_{21} \mathrm{H}_{19} \mathrm{O}_{12}$ & -0.8 & 15.0 & quercetin-hexoside \\
\hline 17 & 9.8 & 595.1429 & + & 147(100), 287(34) & $\mathrm{C}_{30} \mathrm{H}_{27} \mathrm{O}_{13}$ & 2.9 & 24.8 & $\begin{array}{c}\text { kaempferol-hexoside- } \\
\text { deoxyhexoside-coumaric } \\
\text { acid }\end{array}$ \\
\hline 18 & 10.3 & 593.1854 & + & $285(100), 447(6)$ & $\mathrm{C}_{28} \mathrm{H}_{33} \mathrm{O}_{14}$ & 1.8 & 9.2 & $\begin{array}{l}\text { unidentified-hexoside- } \\
\text { deoxyhexoside } \\
\text { (flavonoid) }\end{array}$ \\
\hline 19 & 13.0 & 327.2174 & - & $327(100), 211(9)$ & $\mathrm{C}_{18} \mathrm{H}_{31} \mathrm{O}_{5}$ & 1.0 & 1.5 & unidentified \\
\hline 20 & 14.2 & 329.2327 & - & $329(100), 211(17)$ & $\mathrm{C}_{18} \mathrm{H}_{33} \mathrm{O}_{5}$ & 2.1 & 3.2 & unidentified \\
\hline 21 & 14.6 & 289.2375 & + & $\begin{array}{c}235(100), 253(74) \\
217(64), 135(19) \\
161(14)\end{array}$ & $\mathrm{C}_{16} \mathrm{H}_{33} \mathrm{O}_{4}$ & -0.7 & 8.0 & unidentified \\
\hline
\end{tabular}


Table 8. Compounds identified in the ribwort plantain (Plantago lanceolata L.) extract using UHPLC-QTOF-MS/MS. Compound numbers correspond to those indicated in Figure 2.

\begin{tabular}{|c|c|c|c|c|c|c|c|c|}
\hline Peak & $\begin{array}{c}\mathrm{RT} \\
{[\mathrm{min}]}\end{array}$ & $\begin{array}{l}\text { Molecular Ion } \\
{[\mathrm{M}-\mathrm{H}]^{-}}\end{array}$ & $\begin{array}{c}\text { Ion } \\
\text { Mode }\end{array}$ & $\begin{array}{c}\text { MS/MS } \\
\text { Fragments }\end{array}$ & Formula & $\begin{array}{c}\text { Error } \\
\text { [ppm] }\end{array}$ & mSigma & Tentative Identification \\
\hline 1 & 1.4 & 373.1142 & - & $\begin{array}{c}373(100), 211(92) \\
123(24)\end{array}$ & $\mathrm{C}_{16} \mathrm{H}_{21} \mathrm{O}_{10}$ & -0.4 & 8.3 & geniposidic acid \\
\hline 2 & 2.1 & 461.1672 & - & $461(100), 315(3)$ & $\mathrm{C}_{20} \mathrm{H}_{29} \mathrm{O}_{12}$ & -1.7 & 7.5 & decaffeoylacteoside \\
\hline 3 & 3.3 & 451.2192 & - & $\begin{array}{c}405(100), 179(8) \\
243(8), 167(7)\end{array}$ & $\mathrm{C}_{20} \mathrm{H}_{35} \mathrm{O}_{11}$ & -1.7 & 4.6 & caryoptoside isomer \\
\hline 4 & 3.9 & 813.1363 & - & $\begin{array}{l}\text { 285(100), 637(23), } \\
\text { 351(18), 461(15), }\end{array}$ & $\mathrm{C}_{33} \mathrm{H}_{33} \mathrm{O}_{24}$ & 0.6 & 7.9 & $\begin{array}{l}\text { luteolin-tri-oxyhexide } \\
\text { isomer }\end{array}$ \\
\hline 5 & 4.1 & 637.1044 & - & 285(100), 351(89) & $\mathrm{C}_{27} \mathrm{H}_{25} \mathrm{O}_{18}$ & 0.4 & 20.9 & $\begin{array}{l}\text { luteolin-di-oxyhexide } \\
\text { isomer }\end{array}$ \\
\hline 6 & 4.6 & 639.1941 & - & $\begin{array}{c}\text { 639(100), 621(42), } \\
\text { 161(28), 135(9) } \\
447(7)\end{array}$ & $\mathrm{C}_{29} \mathrm{H}_{35} \mathrm{O}_{16}$ & -1.6 & 15.8 & $\begin{array}{c}\text { unidentified } \\
\text { phenylethanoid }\end{array}$ \\
\hline 7 & 4.7 & 639.1933 & - & $\begin{array}{c}\text { 639(100), 621(59), } \\
\text { 161(28), 133(16), } \\
475(11)\end{array}$ & $\mathrm{C}_{29} \mathrm{H}_{35} \mathrm{O}_{16}$ & -0.4 & 11.7 & $\begin{array}{c}\text { unidentified } \\
\text { phenylethanoid }\end{array}$ \\
\hline 8 & 5.4 & 637.1046 & - & 285(100), 461(44) & $\mathrm{C}_{27} \mathrm{H}_{25} \mathrm{O}_{18}$ & 0.0 & 7.6 & $\begin{array}{l}\text { luteolin-di-oxyhexoside } \\
\text { isomer }\end{array}$ \\
\hline 9 & 5.7 & $\begin{array}{l}461.0721 \\
989.1849 \\
639.1935\end{array}$ & $\begin{array}{l}- \\
- \\
-\end{array}$ & $\begin{array}{c}285(100) \\
285(100), 461(12), \\
813(8) \\
639(100), 285(64), \\
161(41), 477(32), \\
461(21)\end{array}$ & $\begin{array}{l}\mathrm{C}_{21} \mathrm{H}_{17} \mathrm{O}_{12} \\
\mathrm{C}_{43} \mathrm{H}_{41} \mathrm{O}_{27} \\
\mathrm{C}_{29} \mathrm{H}_{35} \mathrm{O}_{16}\end{array}$ & $\begin{array}{c}1.1 \\
-0.8 \\
-0.7\end{array}$ & $\begin{array}{c}6.8 \\
43.6 \\
8.1\end{array}$ & $\begin{array}{l}\text { luteolin-oxyhexoside } \\
\text { isomer } \\
\text { unidentified luteolin } \\
\text { derivative (flavonoid) } \\
\text { unidentified } \\
\text { phenylethanoid }\end{array}$ \\
\hline 10 & 6.3 & $\begin{array}{l}545.2231 \\
477.1400 \\
755.2407\end{array}$ & $\begin{array}{l}- \\
- \\
-\end{array}$ & $\begin{array}{c}545(100), 337(14) \\
235(10), 193(8) \\
477(100), 161(22), \\
133(11), 315(9) \\
179(2) \\
755(100), 161(21), \\
179(10), 593(10), \\
135(8)\end{array}$ & $\begin{array}{l}\mathrm{C}_{25} \mathrm{H}_{37} \mathrm{O}_{13} \\
\mathrm{C}_{23} \mathrm{H}_{25} \mathrm{O}_{11} \\
\mathrm{C}_{34} \mathrm{H}_{43} \mathrm{O}_{19}\end{array}$ & $\begin{array}{c}1.5 \\
0.4 \\
-0.4\end{array}$ & $\begin{array}{c}10.4 \\
9.5 \\
12.5\end{array}$ & $\begin{array}{c}\text { unidentified } \\
\text { calceolarioside A isomer } \\
\text { forsythoside isomer }\end{array}$ \\
\hline 11 & 6.5 & $\begin{array}{l}623.1980 \\
639.1930\end{array}$ & - & $\begin{array}{c}623(100), 161(26) \\
461(12) \\
639(100), 161(30) \\
477(13), 133(6)\end{array}$ & $\begin{array}{l}\mathrm{C}_{29} \mathrm{H}_{35} \mathrm{O}_{15} \\
\mathrm{C}_{29} \mathrm{H}_{35} \mathrm{O}_{16}\end{array}$ & $\begin{array}{l}0.2 \\
0.2\end{array}$ & $\begin{array}{c}5.6 \\
16.6\end{array}$ & $\begin{array}{c}\text { verbascoside isomer } \\
\text { unidentified } \\
\text { phenylethanoid }\end{array}$ \\
\hline 12 & 6.9 & $\begin{array}{l}445.0779 \\
755.2401\end{array}$ & - & $\begin{array}{c}269(100) \\
755(100), 161(24), \\
593(11), 133(8)\end{array}$ & $\begin{array}{l}\mathrm{C}_{21} \mathrm{H}_{17} \mathrm{O}_{11} \\
\mathrm{C}_{34} \mathrm{H}_{43} \mathrm{O}_{19}\end{array}$ & $\begin{array}{c}-0.6 \\
0.4\end{array}$ & $\begin{array}{l}24.9 \\
19.4\end{array}$ & $\begin{array}{l}\text { apigenin-oxyhexoside } \\
\text { forsythoside isomer }\end{array}$ \\
\hline 13 & 7.1 & 623.1987 & - & $\begin{array}{c}\text { 623(100), 161(17) } \\
461(7), 133(5)\end{array}$ & $\mathrm{C}_{29} \mathrm{H}_{35} \mathrm{O}_{15}$ & -0.9 & 7.0 & verbascoside isomer \\
\hline 14 & 7.5 & 475.0877 & - & 274(100), 299(73) & $\mathrm{C}_{22} \mathrm{H}_{19} \mathrm{O}_{12}$ & 1.0 & 7.5 & $\begin{array}{l}\text { kaempferide- } \\
\text { oxyhexoside }\end{array}$ \\
\hline 15 & 8.0 & 637.2138 & - & $\begin{array}{c}\text { 637(100), 461(59) } \\
175(41)\end{array}$ & $\mathrm{C}_{30} \mathrm{H}_{37} \mathrm{O}_{15}$ & 0.0 & 15.9 & leucoseptoside A isomer \\
\hline 16 & 8.0 & 621.1826 & & $\begin{array}{c}\text { 321(100), 323(21) } \\
179(18), 487(14)\end{array}$ & $\mathrm{C}_{29} \mathrm{H}_{33} \mathrm{O}_{15}$ & -0.2 & 4.8 & unidentified \\
\hline 17 & 8.4 & 629.2674 & - & $\begin{array}{c}\text { 583(100), 421(21) } \\
451(13), 289(9)\end{array}$ & $\mathrm{C}_{26} \mathrm{H}_{45} \mathrm{O}_{17}$ & -1.9 & 5.9 & unidentified \\
\hline 18 & 10.5 & 651.2304 & - & $\begin{array}{c}\text { 651(100), 175(24), } \\
\text { 160(12), 193(7), } \\
475(5)\end{array}$ & $\mathrm{C}_{31} \mathrm{H}_{39} \mathrm{O}_{15}$ & -1.5 & 12.5 & unidentified \\
\hline
\end{tabular}


Table 9. Compounds identified in the marshmallow (Althaea L.) extract using UHPLC-QTOF-MS/MS. Compound numbers correspond to those indicated in Figure 2.

\begin{tabular}{|c|c|c|c|c|c|c|c|c|}
\hline Peak & $\begin{array}{c}\text { RT } \\
{[\mathrm{min}]}\end{array}$ & $\begin{array}{l}\text { Molecular Ion } \\
{[\mathrm{M}-\mathrm{H}]^{-}}\end{array}$ & $\begin{array}{l}\text { Ion } \\
\text { Mode }\end{array}$ & $\begin{array}{c}\text { MS/MS } \\
\text { Fragments }\end{array}$ & Formula & $\begin{array}{l}\text { Error } \\
{[\mathrm{ppm}]}\end{array}$ & mSigma & Tentative Identification \\
\hline 1 & 1.4 & 326.1246 & - & $\begin{array}{c}\text { 164(100), 236(26) } \\
147(20)\end{array}$ & $\mathrm{C}_{15} \mathrm{H}_{20} \mathrm{NO}_{7}$ & -0.2 & 16.1 & phenylalanyl-hexoside \\
\hline 2 & 2.5 & 417.1043 & - & 417(100), 152(8) & $\mathrm{C}_{17} \mathrm{H}_{21} \mathrm{O}_{12}$ & -1.0 & 4.6 & gentisic acid-dipentoside \\
\hline 3 & 2.8 & $\begin{array}{l}179.0357 \\
361.0966\end{array}$ & $\begin{array}{l}- \\
-\end{array}$ & $\begin{array}{c}179(100), 135(62) \\
361(100), 281(13) \\
171(7)\end{array}$ & $\begin{array}{c}\mathrm{C}_{9} \mathrm{H}_{7} \mathrm{O}_{4} \\
\mathrm{C}_{24} \mathrm{H}_{13} \mathrm{~N}_{2} \mathrm{O}_{2}\end{array}$ & $\begin{array}{l}-4.1 \\
4.7\end{array}$ & $\begin{array}{l}41.7 \\
55.9\end{array}$ & $\begin{array}{l}\text { caffeic acid } \\
\text { unidentified }\end{array}$ \\
\hline 4 & 3.5 & 227.0568 & - & $\begin{array}{c}227(100), 139(22) \\
165(9)\end{array}$ & $\mathrm{C}_{10} \mathrm{H}_{11} \mathrm{O}_{6}$ & -3.1 & 9.2 & unidentified \\
\hline 5 & 3.7 & 623.0037 & - & & $\mathrm{C}_{17} \mathrm{H}_{19} \mathrm{O}_{23} \mathrm{~S}$ & 1.0 & 32.2 & $\begin{array}{c}\text { flavonoid } \\
\text { disulfo-hexoside }\end{array}$ \\
\hline 6 & 5.0 & 193.0479 & + & $\begin{array}{c}\text { 193(100), 134(36), } \\
\text { 178(36), 191(10) }\end{array}$ & $\mathrm{C}_{10} \mathrm{H}_{9} \mathrm{O}_{4}$ & 8.3 & 9.7 & scopoletin isomer \\
\hline 7 & 5.2 & 425.0562 & - & $\begin{array}{c}425(100), 297(88) \\
315(86), 241(75) \\
327(73)\end{array}$ & $\mathrm{C}_{14} \mathrm{H}_{17} \mathrm{O}_{15}$ & 2.5 & 38.1 & unidentified \\
\hline 8 & 5.9 & 636.9843 & - & & $\mathrm{C}_{17} \mathrm{H}_{16} \mathrm{O}_{24} \mathrm{~S}$ & -1.7 & 12.3 & flavonoid sulfo-glycoside \\
\hline 9 & 6.1 & 733.0950 & - & & $\mathrm{C}_{28} \mathrm{H}_{29} \mathrm{O}_{21} \mathrm{~S}$ & -3.1 & 37.4 & flavonoid sulfo-glycoside \\
\hline 10 & 6.3 & 541.0317 & - & $\begin{array}{c}254(100), 285(33) \\
175(9)\end{array}$ & $\mathrm{C}_{21} \mathrm{H}_{17} \mathrm{O}_{15} \mathrm{~S}$ & -4.3 & 12.5 & theograndin I isomer \\
\hline 11 & 6.4 & 433.1521 & - & $\begin{array}{l}\text { 433(100), 403(86), } \\
311(76), 299(58)\end{array}$ & $\mathrm{C}_{22} \mathrm{H}_{25} \mathrm{O}_{9}$ & -3.9 & 5.5 & unidentified \\
\hline 12 & 6.7 & $\begin{array}{l}527.0522 \\
639.1223\end{array}$ & $\begin{array}{l}- \\
-\end{array}$ & $\begin{array}{c}285(100), 527(4), \\
213(4), 447(4) \\
301(100), 371(25), \\
299(24), 459(22)\end{array}$ & $\begin{array}{l}\mathrm{C}_{21} \mathrm{H}_{19} \mathrm{O}_{14} \mathrm{~S} \\
\mathrm{C}_{27} \mathrm{H}_{27} \mathrm{O}_{18}\end{array}$ & $\begin{array}{l}-4.1 \\
-3.1\end{array}$ & $\begin{array}{l}40.7 \\
62.4\end{array}$ & $\begin{array}{l}\text { flavonoid sulfo-glycoside } \\
\text { unidentified- } \\
\text { deoxyhexoside-hexoside } \\
\text { (flavonoid) }\end{array}$ \\
\hline 13 & 7.2 & 524.2881 & - & $524(100), 362(50)$ & $\mathrm{C}_{27} \mathrm{H}_{42} \mathrm{NO}_{9}$ & -3.1 & 25.3 & unidentified \\
\hline 14 & 7.3 & & & & & & & unidentified \\
\hline 15 & 7.6 & 557.0266 & - & $\begin{array}{l}\text { 301(100), 254(77), } \\
\text { 315(58), 271(42) }\end{array}$ & $\mathrm{C}_{21} \mathrm{H}_{17} \mathrm{O}_{16} \mathrm{~S}$ & -4.2 & 21.0 & theograndin II isomer \\
\hline 16 & 8.5 & 555.0464 & & $\begin{array}{c}254(100), 284(30) \\
299(24), 175(7)\end{array}$ & $\mathrm{C}_{22} \mathrm{H}_{19} \mathrm{O}_{15} \mathrm{~S}$ & -2.5 & 9.6 & $\begin{array}{c}\text { flavonoid } \\
\text { sulfo-oxyhexoside }\end{array}$ \\
\hline 17 & 8.9 & 541.0672 & - & $\begin{array}{c}299(100), 284(68) \\
461(6)\end{array}$ & $\mathrm{C}_{22} \mathrm{H}_{21} \mathrm{O}_{14} \mathrm{~S}$ & -2.7 & 14.1 & flavonoid sulfo-hexoside \\
\hline 18 & 8.9 & 541.0310 & - & $\begin{array}{c}285(100), 254(67) \\
461(22)\end{array}$ & $\mathrm{C}_{21} \mathrm{H}_{17} \mathrm{O}_{15} \mathrm{~S}$ & -3.1 & 34.2 & $\begin{array}{c}\text { flavonoid } \\
\text { sulfo-oxyhexoside }\end{array}$ \\
\hline 19 & 9.1 & 571.0411 & - & $\begin{array}{c}\text { 300(100), 254(79), } \\
315(46), 491(23)\end{array}$ & $\mathrm{C}_{22} \mathrm{H}_{19} \mathrm{O}_{16} \mathrm{~S}$ & -2.0 & 24.2 & $\begin{array}{c}\text { flavonoid } \\
\text { sulfo-oxyhexoside }\end{array}$ \\
\hline 20 & 9.4 & 307.0731 & - & $\begin{array}{c}\text { 233(100), 205(76) } \\
263(75), 191(9)\end{array}$ & $\mathrm{C}_{17} \mathrm{H}_{11} \mathrm{~N}_{2} \mathrm{O}_{4}$ & -2.2 & 2.1 & unidentified \\
\hline 21 & 10.5 & 475.0896 & - & & $\mathrm{C}_{22} \mathrm{H}_{19} \mathrm{O}_{12}$ & -3.1 & 56.1 & unidentified \\
\hline 22 & 11.4 & 555.0469 & - & $\begin{array}{c}\text { 254(100), 284(80), } \\
\text { 299(71), 475(35), } \\
175(40)\end{array}$ & $\mathrm{C}_{22} \mathrm{H}_{19} \mathrm{O}_{15} \mathrm{~S}$ & -3.4 & 1.8 & $\begin{array}{c}\text { flavonoid } \\
\text { sulfo-oxyhexoside }\end{array}$ \\
\hline 23 & 18.1 & 311.2241 & - & & $\mathrm{C}_{18} \mathrm{H}_{31} \mathrm{O}_{4}$ & -4.2 & 4.5 & unidentified \\
\hline 24 & 19.3 & 459.2037 & - & & $\mathrm{C}_{25} \mathrm{H}_{31} \mathrm{O}_{8}$ & -2.8 & 24.7 & unidentified \\
\hline 25 & 19.8 & 459.2030 & - & & $\mathrm{C}_{25} \mathrm{H}_{31} \mathrm{O}_{8}$ & -1.3 & 56.6 & unidentified \\
\hline
\end{tabular}




\section{Discussion}

\subsection{Antimicrobial Activity of Plant Extracts}

Our experiments showed that with the increase in dose the tested plant extracts gradually limited the acidifying activity of the tested LAB, but did not limit the viability of these cells. This finding is interesting considering the necessity to keep the LAB cells alive and maintain them biologically active in fermented milk drinks throughout the declared shelf life. Perhaps, these plant extracts could be used in the dose range studied to stop the activity of starter bacteria and consequently prevent acidification of the fermented milk beverages with the cultures used in this study. It should be noted that there are no studies to date in this regard.

Various spices and herbal extract may affect activity or vitality of lactic acid bacteria, and this phenomenon depend on the genus, species and even sometimes strain of lactic bacteria, the species of herbs and the method of obtaining the extract from them. It is known from research that some LABs have the ability to degrade certain phenolic compounds found in food, including those with high antioxidant activity [33]. Polyphenol-resistant bacteria have the ability to metabolize polyphenol compounds, depending on their chemical structure (substitutions in the phenolic ring) and concentration. Lactobacillus plantarum has been described to exhibit several enzymatic activities, such as that of tannase, phenolic acid decarboxylase (PAD), and benzyl alcohol dehydrogenase which can degrade some phenolic compounds [33]. Only limited studies have analyzed the influence of phenolic compounds on the growth and viability of other LAB species $[34,35]$.

We did not observe any negative or positive effect of the added extracts on the viability of lactobacilli cells during the fermentation process. This is in line with the results reported by Otaibi and Demerdash [36], who showed that appropriately selected portions of sage extracts resulted in better survival of yogurt bacterial cells, while El-Nawawy et al. [37] indicated a beneficial effect of extracts on the multiplication of bacteria in yogurt. An increase was observed in the numbers of $S$. thermophilus cells than L. delbrueckii subsp. bulgaricus. However, after digestion, the number of Streptococcus cells decreased by an average of $1 \log$ cycle, while the population of Lactobacillus cells remained stable. This is also confirmed by the studies of Zaręba et al. [38] and Ziarno and Margol [39], which proves that $\mathrm{LAB}$ poorly tolerate digestive juices, but their survival rate is highly dependent on the culture used. The results obtained in this study thus confirm that the viability of LAB in selected starter cultures can be maintained at a fairly high level, even under such drastic conditions as digestive juices.

There are studies on the antimicrobial effect of plant extracts available in the literature. The antimicrobial activity of plant extracts investigated in this work is often the subject of scientific research [40], but only a few concern the effect of extracts on the activity or population of selected LABs [36]. Due to the lack of comprehensive information on the effect of selected plant extracts on LABs, it is worth taking a brief look at the effect of other plant additives on the bacteria in question. Fortunately, a few reports are found in the literature on the beneficial or inhibitory effects of different plant additives on LABs [41-46].

Among the few publications dealing with the effects of valerian ( $V$. officinalis $L$.) on bacteria, there are some reports on its antibacterial activity [47]. However, no data are available regarding the effect of purge on LABs. Sage (S. officinalis L.) oils have proven antibacterial and antifungal properties [40,48-50]. Their effect on yogurt bacteria has also been studied proving that the appropriate portions of these additives do not inhibit the growth of yogurt bacteria, and on the contrary, they may have a positive effect on their survival and increase their number during storage [36]. This was also confirmed by the results of our research. Moreover, El-Nawawy et al. [37] reported a beneficial effect of sage extracts on the population of S. thermophilus and L. bulgaricus. On the other hand, Hołderna-Kedzia and Kedzia [51] showed a negative effect of these extracts on Lactobacillus acidophilus ATCC 4356, Lactobacillus casei ATCC 393, Lactobacillus rhamnosus Hansen 1968, Bifidobacterium bifidum ATCC 35914, S. thermophilus ATCC 14485, and Saccharomyces boulardii SB48 ATCC-MYA-796. The antibacterial effect of chamomile (M. chamomilla L.) extracts 
has been described in many studies [49,51-53]. However, there are no scientific reports on the effect of chamomile extract on LABs. Several researchers have studied the activity of cistus (Cistus L.) extract against pathogenic fungi and bacteria [54-57]. Few reports even indicate that linden blossom has a slight antibacterial effect (Tilia L.) [58,59], but there are no data on its influence on LABs. Ethanol and methanol extracts of ribwort plantain (P. lanceolata L.) have already been tested against the strains of Staphylococcus aureus, Staphylococcus epidermidis, Bacillus subtilis, Bacillus cereus, Proteus mirabilis, Pseudomonas aeruginosa, Escherichia coli, Enterobacter aerogenes, Klebsiella pneumoniae, Candida albicans, and Candida tropicalis and found to be active against these bacteria [60-63].

For marshmallow (Althaea L.) extracts, it has been shown that the extracts inhibited the growth of bacteria such as Pseudomonas fluorescens, P. aeruginosa, Bordetella bronchiseptica, S. aureus, S. epidermidis, Micrococcus luteus, Enterococcus faecalis, B. subtilis, B. cereus, Aspergillus niger, C. albicans, and Saccharomyces cerevisiae [64,65]. It was also showed that marshmallow extracts did not have any inhibitory effect on E. coli, K. pneumoniae, or Serratia marcescens [64]. However, the effect of these extracts on LABs has not yet been studied.

\subsection{TPC and Antioxidant Capacities of Plant Extracts}

It is reported that the metabolites present in plant extracts, including phenolic compounds, are responsible for their various biological activities, such as total phenolic content and antioxidant activity $[20,22,66-68]$. Therefore, it is worth analyzing the research proving the antioxidant activity of selected herbal extracts. Phenolic compounds are widespread in the world of plants. Based on the structure of the carbon skeleton, phenolic compounds can be divided into phenolic acids and flavonoids.

Wang et al. [69] and Şen and Mat [66] indicated that sesquiterpenes, iridoids, flavonoids, and alkaloids were isolated from valerian ( $V$. officinalis L.) extracts. Katsarova et al. [67] showed the lowest antioxidant activity for valerian extracts among the eight tested plant extracts (V. officinalis, Melissa officinalis, Crataegus monogyna, Hypericum perforatum, Serratula coronata, and their two combinations): oxygen radical absorbing capacity— $820.5 \pm 21.9 \mu \mathrm{mol} \mathrm{TE} / \mathrm{g}$; hydroxyl radical averting capacity-381.6 $\pm 14.0 \mu \mathrm{mol}$ GAE/g; and polyphenolic content- $43.36 \pm 1.3 \mathrm{mg} / \mathrm{g}$. In our study, the valerian extract showed slightly less TPC, expressed as mg GAE/g extract, compared to the above value, despite the fact that its chemical composition included a large diversity of ingredients with antioxidant properties. Furthermore, compared to the other tested plant extracts, its antioxidant capacities were lower.

The crude extract of sage (S. officinalis L.) studied by Koşar et al. [70] contained hydroxybenzoic acids, hydroxycinnamic acids, flavonoids, and diterpenoids, in addition to caffeic acid, carnosic acid, luteolin, luteolin-7-O-glucoside, and rosmarinic acid. However, the composition of the plant extract may vary depending on the method used for extraction [5,71-73]. The sage extract obtained by Houghton [5] also contained cyclic monoterpenes, such as 1,8-cineol, $\alpha$-pinene, and camphor. The crude extract and subfractions demonstrated varying degrees of antioxidant capacity. Rosmarinic acid and abietane diterpenes were thought to be responsible for the potent scavenging properties of Salvia taxa [74,75]. Our research confirmed a good correlation between the high TPC in the sage extract and its high antioxidant capacities. Modern analytical methods based on semipreparative HPLC, high-resolution MS, nuclear magnetic resonance spectroscopy, infrared spectroscopy, and single-crystal X-ray diffraction were used to analyze the chemical composition of different sage extracts obtained from the areal parts. A substantial difference was found between the composition of sage flower $\mathrm{CO} 2$ extract and that of sage flower resin extract [76]. In addition to the known compounds, novel compounds were identified in sage flowers. Among these, some were preidentified in our research, namely danshensu, caffeic acid, rosmarinic acid, luteolin, and apigenin.

The basic active substances of chamomile (M. chamomilla L.) include essential oils, flavonoids, coumarins, sesquiterpenes, polyacetylenes, spiroether, choline, mucus compounds, vitamin $C$, and mineral salts. Chamomile oil contains compounds that have 
a specific healing effect, such as antiallergic chamazulene and bisabolol and its oxides. Many bioactive phenolic compounds, including herniarin and umbelliferone (coumarin), chlorogenic acid and caffeic acid (phenylpropanoids), apigenin, apigenin-7-O-glucoside, luteolin and luteolin-7-O-glucoside (flavones), quercetin and rutin (flavonols), and naringenin (flavanone), have been found in chamomile extract [77-79]. Among flavonoids, apigenin is the most promising due to its multiple therapeutic functions. It exists in the form of various glycosides or in very small quantities as free apigenin. The method of extraction not only determines the chemical composition of chamomile extract but also its antioxidant activity [72]. Flavonoids represent the major fraction of water-soluble components in chamomile [71]. Their values in chamomile aqueous extract were established as follows: quercetin equivalent per gram of extract $-27.65 \pm 0.007 \mu \mathrm{g}$; GAE per gram of extract $-146.97 \pm 0.046 \mu \mathrm{g}$, and tannic acid equivalent per gram of extract-132.22 $\pm 0.023 \mu \mathrm{g}$. In contrast, analysis of chamomile extract containing many flavonoids and numerous organic acids and their derivatives in this study revealed the low TPC compared to other tested plant extracts tested, as well as one of the lowest antioxidant capacities.

Viapiana et al. [9] determined the content of phenolic acids and flavonoids in cistus (Cistus L.) extracts. Their results revealed that aqueous cistus extracts were richer in phenolic compounds and showed strong antioxidant activities. The total amount of polyphenols in the leaf, stalk, and bud extracts of Cistus incanus determined by Dimcheva and Karsheva [73] varied between 36.26 and $115.32 \mathrm{mg}$ GAE/g dry weight ( $\mathrm{dw})$, depending on the time of extraction. After $180 \mathrm{~min}$ of $C$. incanus extraction, the phenolic content was slightly higher than that observed in our study. Such result has also been shown by other studies. Researchers showed that the place of origin is the main factor differentiating the antibacterial activities of cistus samples. Dimcheva et al. [10] found catechins, flavonoids, and gallic and vanillic acids in Cistus extracts. The compounds identified by Gori et al. [80] in crude ethanolic leaf extract of $C$. incanus included gallic acid derivatives, condensed tannins, and flavonol glycosides. In total, 19 compounds were identified based on the fragmentation of individual polyphenols and by comparing their retention times and UV/Vis spectra with authentic standards. As in our research, the presence of epicatechin, myricetin hexoside, and quercetin pentoside was found in the C. incanus extract by Gori et al. [80]. In our study, the Cistus extract had the highest TPC among the tested plant extracts, and thus showed the highest antioxidant capacities. Its composition included many organic acids and their derivatives, as well as flavonoids such as catechin, quercetin, kaempferol, and myricetin.

Several studies have been performed on linden blossom (Tilia L.) extracts. The results revealed the presence of terpenoids, quercetin, and kaempferol derivatives (such as tiliroside), phenolic compounds, esters, aliphatic acids, hydrocarbons, condensed tannins, and a coumarin scopoletin in the extracts [11,16,81,82]. Wissam et al. [83] stated that TPC and the antioxidant activity were determined in the ethanolic extracts of dried linden blossom leaves $(0.3303 \pm 0.0896 \mathrm{mg} / \mathrm{mL}$ calculated as DPPH scavenging activity). In our study, the DPPH value of linden blossom extract was determined as $0.161 \pm 0.007$, but expressed as mmol TE/g extract. This value was the second highest recorded in our experiments. TPC of the linden blossom extract was also one of the largest and resulted from the presence of quercetin, kaempferol, other flavonoids, and their derivatives, as well as many organic acids. In addition, 3,4-dihydroxybenzoic acid, myricetin, rutin, ferulic acid, and 3,4-dihydroxybenzaldehyde were found in abundance in the Tilia tomentosa flower [84]. In turn, the major phenolic compound observed in acetone and methanolic extracts of Tilia argentea was protocatechuic acid. The leaf samples of Tilia species were found to contain many compounds similar to those in flower samples, but each of these samples possessed a unique chemical profile including the percentage and type of flavonoid constituents [85].

Some scientists showed that phenolic compounds, mainly flavonoids and hydroxycinnamic acids, were the main components of hydrophilic ribwort plantain (P. lanceolata L.) extracts [19]. Galvez et al. [20] found that luteolin was biologically important among the flavonoids. According to [17], phenylethanoids, especially Aukubin, are responsible for 
the antimicrobial effects of ribwort plantain extracts. It was reported that these extracts exhibited a strong antioxidant activity [19]. Lukova et al. [68] studied the antioxidant activity of the ethanol extracts of $P$. lanceolata leaves by DPPH scavenging test, CUPRAC (cupric reducing antioxidant capacity) assay, and FRAP assay and established the following values: DPPH-59.04 \pm 0.09\%; CUPRAC $-21.9 \pm 0.58 \mu \mathrm{M}$ TE/g dw; and FRAP-51.85 $\pm 1.54 \mu \mathrm{M}$ $\mathrm{TE} / \mathrm{g}$ dw. The Plantago plantain leaf extract tested in our study was characterized by an average content of TPC expressed as $\mathrm{mg}$ GAE/g of extract, as well as average antioxidant capacities expressed by DPPH, ABTS, or FRAP scavenging ability.

In general, marshmallow (Althaea L.) ethanol extracts show high antioxidant activity, which is due to the presence of active compounds such as flavonoids and mucus polysaccharides [22]. However, this was not confirmed by the results of our research. The leaves of marshmallow contain the coumarin scopoletin, as well as many flavonoids (hypolaetin-8-glucoside, isoquercitrin, kaempferol, caffeic acid, p-coumaric acid, ferulic acid, p-hydroxybenzoic acid, salicylic acid, p-hydroxyphenylacetic acid, vanillic acid) [86,87]. Elmastas et al. [22] indicated the strong total antioxidant activity of ethanolic marshmallow extract. They reported that the marshmallow extract showed effective reducing power, free radical scavenging activity, superoxide anion radical scavenging activity, and metal chelating ability at the same concentration $(50,100$, and $250 \mathrm{mg} / \mathrm{mL}$, respectively). In comparison, the marshmallow extract tested in our study contained only a few antioxidant substances (some flavonoids and organic acids), which resulted in its lower antioxidant capacities.

\section{Conclusions}

This study showed that the addition of herbal extracts from valerian ( $V$. officinalis L.), sage (S. officinalis L.), chamomile (M. chamomilla L.), cistus (Cistus L.), linden blossom (Tilia L.), ribwort plantain (P. lanceolata L.), and marshmallow (Althaea L.) did not inhibit the growth of LAB in fermented milk drinks such as yogurts. In light of the presented results, yogurts enriched with the plant extracts tested in this study can be of interest to customers. However, these herbal extracts should be added in limited amounts because they gradually inhibit the fermentation activity. Now, knowing in what dose range dairy products fermented with the addition of selected herbal extracts can be tested in the future, including storage research. When added at concentrations above $2 \%$ by weight, which probably can be used to prevent overacidification of fermented milk after the fermentation process is complete, herbal extracts from valerian, sage, chamomile, cistus, linden blossom, ribwort plantain, or marshmallow should be tested for the storage stability of fermented milk beverages such as yoghurts containing live lactic acid bacteria.

Nevertheless, due to the antioxidant properties, a slight addition of the herbal extracts containing polyphenols to different kinds of food products can increase the nutritional quality, thus making them functional foods. The microflora of yogurts containing the tested herbal extracts did not die during digestion in model digestive juices, and this amount of bacteria surviving digestion can benefit the health of consumers. Thus, the tested plant extracts had neither an inhibitory nor a stimulating effect on bacteria in the fermented milk samples.

Author Contributions: Conceptualization, M.Z. and M.K. (Mariola Kozłowska); methodology, M.Z., M.K. (Mariola Kozłowska) and I.Ś.; investigation, M.Z., M.K. (Mariola Kozłowska), I.S., M.K. (Mariusz Kowalczyk), S.P., A.S. and B.S.; data curation, M.Z. and B.S.; writing-M.Z., M.K. (Mariola Kozłowska), I.Ś. and B.S.; writing-review and editing, M.Z., M.K. (Mariola Kozłowska), I.Ś. and S.P.; project administration, M.Z. All authors have read and agreed to the published version of the manuscript.

Funding: This work was supported by Polish Ministry of Science and Higher Education with funds of the Warsaw University of Life Sciences WULS-SGGW (Poland).

Institutional Review Board Statement: This study did not involve humans or animals.

Informed Consent Statement: This study did not involve humans. 
Data Availability Statement: Data sharing not applicable.

Conflicts of Interest: The authors declare no conflict of interest.

\section{References}

1. Goun, E.A.; Petrichenko, V.M.; Solodnikov, S.U.; Suhinina, T.V.; Kline, M.A.; Cunningham, G.; Miles, H. Anticancer and antithrombin activity of Russian plants. J. Ethnopharmacol. 2002, 81, 337-342. [CrossRef]

2. O'Hara, M.; Kiefer, D.; Farrell, K.; Kemper, K. A review of 12 commonly used medicinal herbs. Arch. Fam. Med. 1998, 7, 523-536. [CrossRef]

3. Woźniak, M.; Ostrowska, K.; Szymański, Ł.; Wybieralska, K.; Zielińśki, R. Aktywność przeciwrodnikowa ekstraktów szałwii i rozmarynu. Żywność 2009, 4, 133-141, (Abstract in English).

4. Tildesley, N.T.; Kennedy, D.O.; Perry, E.K.; Ballard, C.G.; Savelev, S.A.W.K.; Wesnes, K.A.; Scholey, A.B. Salvia lavandulaefolia (Spanish Sage) enhances memory in health young volunteers. Pharmacol. Biochem. Behav. 2003, 75, 669-674. [CrossRef]

5. Houghton, P.J. Activity and constituents of sage relevant to the potential treatment of symptoms Alzheimer's disease. Herba Gran 2004, 61, 38-43.

6. Khayyal, M.T.; El-Ghazaly, M.A.; Kenawy, S.A.; Seif-El-Nasr, M.; Mahran, L.G.; Kafafi, Y.A.; Okpanyi, S.N. Antiulcerogenic effect of certain plant extracts and their combinations. Arzneimittelforsch 2001,51,545-553.

7. Torrado, S.; Agis, A.; Jimenez, M.E.; Cadorniga, R. Effect of dissolution profile and (-)-alpha-bisabolol on the gastrotoxicity of acetylsalicylic acid. Pharmazie 1995, 50, 141-143. [PubMed]

8. Sebai, H.; Jabri, M.A.; Souli, A.; Rtibi, K.; Selmi, S.; Tebourbi, O.; Sakly, M. Antidiarrheal and antioxidant activities of chamomile (Matricaria recutita L.) decoction extract in rats. J. Ethnopharmacol. 2014, 152, 327-332. [CrossRef]

9. Viapiana, A.; Konopacka, A.; Waleron, K.; Wesolowski, M. Cistus incanus L. commercial products as a good source of polyphenols in human diet. Ind. Crops Prod. 2017, 107, 297-304. [CrossRef]

10. Dimcheva, V.; Kaloyanov, N.; Karsheva, M. The polyphenol composition of Cistus incanus L., Trachystemon orientalis L. and Melissa officinalis L. infusions by HPLC-DAD method. Open J. Anal. Bioanal. Chem. 2019, 3, 031-038. [CrossRef]

11. Arcos, M.L.B.; Cremaschi, G.; Werner, S.; Coussio, J.; Ferraro, G.; Anesini, C. Tília cordata Mill. extracts and scopoletin (isolated compound): Differential cell growth effects on lymphocytes. Phytother. Res. 2006, 20, 34-40. [CrossRef]

12. Hyun Kim, K.; Moon, E.; Kim, S.Y.; Choi, S.U.; Lee, K.R. Lignan constituents of Tilia amurensis and their biological evaluation on antitumor and anti-inflammatory activities. Food. Chem. Toxicol. 2012, 50, 3680-3686.

13. Martinez, A.L.; Gonzalez-Trujano, M.A.; Aguirre-Hernandez, E.; Moreno, J.; Soto-Hernandez, M.; Lopez-Munoz, F.J. Antinociceptive activity of Tilia americana var. mexicana inflorescences and quercetin in the formalin test and in an arthritic pain model in rats. Neuropharmacology 2009, 56, 564-571. [CrossRef]

14. Toker, G.; Küpeli, E.; Memisoğlu, M.; Yesilada, E. Flavonoids with antinociceptive and anti-inflammatory activities from the leaves of Tilia argentea (silver linden). J. Ethnopharmacol. 2004, 95, 393-397. [CrossRef]

15. Aguirre-Hernandez, E.; Martinez, A.L.; Gonzalez-Trujano, M.A.; Moreno, J.; Vibrans, H.; Soto-Hernandez, M. Pharmacological evaluation of the anxiolytic and sedative effects of Tilia americana L. var. mexicana in mice. J. Ethnopharmacol. 2007, 109, 140-145. [CrossRef]

16. Herrera-Ruiz, M.; Román-Ramos, R.; Zamilpa, A.; Tortoriello, J.; Jiménez-Ferrer, J.E. Flavonoids from Tilia americana with anxiolytic activity in plus-maze test. J. Ethnopharmacol. 2008, 118, 312-317. [CrossRef] [PubMed]

17. ESCOP. Monographs 2013: European Scientific Cooperative on Phytotherapy. Plantaginis lanceolatae Folium/Herba, 2nd ed.; Ribwort Plantain Leaf/Herb; European Scientific Cooperative on Phytotherapy (ESCOP): Exeter, UK, 2013; pp. 383-387.

18. Parus, A.; Grys, A. Plantago lanceolata L.-medicinal properties. Post Fit. 2010, 3, 162-165, (Abstract in English).

19. Dalar, A.; Türker, M.; Konczak, I. Antioxidant capacity and phenolic constituents of Malva neglecta Wallr. and Plantago lanceolata L. from Eastern Anatolia Region of Turkey. J. Herbal Med. 2012, 2, 42-51. [CrossRef]

20. Galvez, M.; Martí, C.; Lopez-Lazaro, M.; Cortes, F.; Ayuso, J. Cytotoxic effect of Plantago spp. on cancer cell lines. J. Ethnopharmacol. 2003, 88, 125-130. [CrossRef]

21. Deters, A.; Zippel, J.; Hellenbrand, N.; Pappai, D.; Possemeyer, C.; Hensel, A. Aqueous extracts and polysaccharides from Marshmallow roots (Althea officinalis L.): Cellular internalisation and stimulation of cell physiology of human epithelial cells in vitro. J. Ethnopharmacol. 2010, 127, 62-69. [CrossRef] [PubMed]

22. Elmastas, M.; Ozturk, L.; Gokce, I.; Erenler, R.; Aboul Enein, H.Y. Determination of antioxidant activity of marshmallow flower (Althaea officinalis L.). Anal. Lett. 2004, 37, 1859-1869. [CrossRef]

23. Izzo, A.A.; Di Carlo, G.; Mascolo, N.; Autore, G.; Capasso, F. Antiulcer effect of flavonoids. Role of endogenous PAF. Phytother. Res. 1994, 29, 87-93. [CrossRef]

24. Guerrero, J.A.; Lozano, M.L.; Castillo, J.; Benavente Garcia, O.; Vicente, V.; Rivera, J. Flavonoids inhibit platelet function through binding to the thromboxane A2 receptor. J. Thromb. Haemost. 2005, 3, 369-376. [CrossRef] [PubMed]

25. Kozłowska, M.; Ścibisz, I.; Zaręba, D.; Ziarno, M. Antioxidant properties and effect on lactic acid bacterial growth of spice extracts. CyTA 2015, 13, 573-577. [CrossRef]

26. Kozłowska, M.; Ziarno, M.; Rudzińska, M.; Tarnowska, K.; Majewska, E.; Kowalska, D. Skład chemiczny olejku eterycznego z kolendry i jego wpływ na wzrost wybranych szczepów bakterii kwasu mlekowego. Żywność 2018, 25, 97-111. (Abstract in English) [CrossRef] 
27. Ziarno, M.; Zaręba, D. Effects of milk components and food additives on survival of three bifidobacteria strains in fermented milk under simulated gastrointestinal tract conditions. Microb. Ecol. Health Dis. 2015, 26. [CrossRef] [PubMed]

28. Singleton, V.L.; Rossi, J.A. Colorimetry of total phenolics with phosphomolybdic-phodphotonegstics acid reagents. Am. J. Enol. Viticult. 1965, 16, 144-158.

29. Yen, G.C.; Chen, H.Y. Antioxidant activity of various tea extracts in relation to their antimutagenicity. J. Agric. Food Chem. 1995, 43, 27-32. [CrossRef]

30. Re, R.; Pellegrini, N.; Proteggente, A.; Pannala, A.; Yang, M.; Rice-Evans, C. Antioxidant activity applying an improved ABTS radical cation decolorization assay. Free Rad. Biol. Med. 1999, 26, 1231-1237. [CrossRef]

31. Benzie, I.F.; Strain, J.J. The ferric reducing ability of plasma (FRAP) as a measure of "antioxidant power": The FRAP assay. Anal. Biochem. 1996, 239, 70-76. [CrossRef]

32. Dührkop, K.; Fleischauer, M.; Ludwig, M.; Aksenov, A.A.; Melnik, A.V.; Meusel, M.; Dorrestein, P.C.; Rousu, J.; Böcker, S. SIRIUS 4: A rapid tool for turning tandem mass spectra into metabolite structure information. Nat. Methods 2019, 16, 299-302. [CrossRef]

33. Rodríguez, H.; Curiel, J.A.; Landete, J.M.; de las Rivas, B.; de Felipe, F.L.; Gómez-Cordovés, C.; Mancheño, J.M.; Muñoz, R. Food phenolics and lactic acid bacteria. Int. J. Food Microbiol. 2009, 132, 79-90. [CrossRef] [PubMed]

34. Landete, J.M.; Rodríguez, H.; Curiel, J.A.; de las Rivas, B.; Mancheño, J.M.; Muñoz, R. Gene cloning, expresión, and characterization of phenolic acid decarboxylase from Lactobacillus brevis RM84. J. Ind. Microbiol. Biotechnol. 2010, 37, 617-624. [CrossRef]

35. Takagaki, A.; Nanjo, F. Metabolism of (-)-epigallocatechin gallate by rat intestinal flora. J. Agric. Food Chem. 2010, 58, $1313 \mathrm{e} 1321$. [CrossRef]

36. Otaibi, M.A.; Demerdash, H.E. Improvement of the quality and shelf life of concentrated yoghurt (labneh) by the addition of some essential oils. Afr. J. Microbiol. Res. 2008, 2, 156-161.

37. El-Nawawy, M.A.; El-Kenany, Y.M.; Abd El-Ghaffar, E.A. Effect of some herb plants on the use of yoghurt culture. Ann. Agric. Sci. 1998, 7, 15-17.

38. Zaręba, D.; Ziarno, M.; Strzelczyk, B. Przeżywalność bakterii fermentacji mlekowej w warunkach modelowych jelita cienkiego. Żywność 2008, 15, 197-205, (Abstract in English).

39. Ziarno, M.; Margol, B. Research into the Ability of Some Selected Starter Lactic Acid Bacteria (Slab) to Survive in a Model Gastric Juice and Cholesterol Binding Under Those These Conditions. Żywność 2007, 6, 304-314, (Abstract in English).

40. Al-Turki, A.I.; El-Ziney, M.G.; Abdel-Salam, A.M. Chemical and anti-bacterial characterization of aqueous extracts of oregano, marjoram, sage and licorice and their application in milk and labneh. J. Food Agric. Environ. 2008, 6, 39-44.

41. Nes, I.F.; Skjelkvale, R. Effect of natural spices and oleoresins on Lactobacillus plantarum in the fermentation of dry sausage. J. Food Sci. 1982, 47, 1618-1625. [CrossRef]

42. Kivanç, M.; Akgül, A.; Doğan, A. Inhibitory and stimulatory effects of cumin, oregano and their essential oils on growth and acid production of Lactobacillus plantarum and Leuconostoc mesenteroides. Int. J. Food Microbiol. 1991, 13, 81-85. [CrossRef]

43. Michael, M.; Phebus, R.K.; Schmidt, K.A. Impact of a plant extract on the viability of Lactobacillus delbrueckii ssp. bulgaricus and Streptococcus thermophilus in nonfat yogurt. Int. Dairy J. 2010, 20, 665-672. [CrossRef]

44. Yangmei, Q.; Jiang, W.; Hou, H.; Zhang, H. Application of Plant Green Juice Powder in Maintaining Stability of Viable Count of Yoghourt in Shelf Life. Patent No. CN 102106386 B, 22 March 1991.

45. China, R.; Mukherjee, S.; Sen, S.; Bose, S.; Datta, S.; Koley, H.; Dhar, P. Antimicrobial activity of Sesbania grandiflora flower polyphenol extracts on some pathogenic bacteria and growth stimulatory effect on the probiotic organism Lactobacillus acidophilus. Microbiol. Res. 2012, 167, 500-506. [CrossRef] [PubMed]

46. Dimitrova, M.; Ivanov, G.; Mihalev, K.; Slavchev, A.; Ivanova, I.; Vlaseva, R. Investigation of antimicrobial activity of polyphenolenriched extracts against probiotic lactic acid bacteria. Food Sci. Appl. Biotechnol. 2019, 2, 67-73. [CrossRef]

47. Letchamo, W.; Ward, W.; Heard, B.; Heard, D. Essential oil of Valeriana officinalis L. cultivars and their antimicrobial activity as influenced by harvesting time under commercial organic cultivation. J. Agric. Food Chem. 2004, 52, 3915-3919. [CrossRef] [PubMed]

48. Hać-Szymańczuk, E.; Lipińska, E.; Błażejak, S.; Bieniak, K. Ocena aktywności przeciwbakteryjnej szałwii lekarskiej (Salvia officinalis L.). Brom. Chem. Toksykol. 2011, 44, 667-672, (Abstract in English).

49. Kędzia, A.; Dera-Tomaszewska, B.; Ziółkowska-Klinkosz, M.; Kędzia, A.W.; Wojtaszek-Słomińska, A.; Czernecka, B. Działanie olejku szałwiowego (Oleum Salviae lavandulaefoliae) na bakterie tlenowe izolowane z jamy ustnej, dróg oddechowych i przewodu pokarmowego. Post. Fitoter. 2011, 1, 238-242, (Abstract in English).

50. Gniewosz, M.; Kraśniewska, K.; Węglarz, Z.; Przybył, J.L. Porównanie przeciwdrobnoustrojowej aktywności etanolowego i wodnego ekstraktu z szałwii lekarskiej (Salvia officinalis L.). Brom. Chem. Toksykol. 2012, 45, 743748, (Abstract in English).

51. Hołderna-Kędzia, E.; Kędzia, B. Działanie preparatów pochodzenia roślinnego na drobnoustroje probiotyczne. Post. Fitoter. 2012, 1, 72-77, (Abstract in English).

52. Grys, A.; Kania, M.; Baraniak, J. Rumianek-Pospolita roślina zielarska o różnorodnych właściwościach biologicznych i leczniczych. Post. Fitoter. 2014, 1, 90-93, (Abstract in English).

53. Roby, M.H.H.; Sarhan, M.A.; Selim, K.A.H.; Khalel, K.I. Antioxidant and antimicrobial activities of essential oil and extracts of fennel (Foeniculum vulgare L.) and chamomile (Matricaria chamomilla L.). Ind. Crops Prod. 2013, 44, 437-445. [CrossRef] 
54. Ustün, O.; Ozçelik, B.; Akyön, Y.; Abbasoglu, U.; Yesilada, E. Flavonoids with anti-Helicobacter pylori activity from Cistus laurifolius leaves. J. Ethnopharmacol. 2006, 108, 457-461. [CrossRef]

55. Hutschenreuther, A.; Birkemeyer, C.; Grötzinger, K.; Straubinger, R.K.; Rauwald, H.W. Growth inhibiting activity of volatile oil from Cistus creticus L. against Borrelia burgdorferi in vitro. Pharmazie 2010, 65, 290-295. [PubMed]

56. Tomás, M.L.; Morales-Soto, A.; Barrajón-Catalán, E.; Roldán-Segura, C.; Segura-Carretero, A.; Micol, V. Correlation between the antibacterial activity and the composition of extracts derived from various Spanish Cistus species. Food. Chem. Toxicol. 2013, 55, 313-322. [CrossRef]

57. Rebaya, A.; Belghith, S.I.; Hammrouni, S.; Maaroufi, A.; Ayadi, M.T.; Chérif, J.K. Antibacterial and Antifungal Activities of Ethanol Extracts of Halimium halimifolium, Cistus salviifolius and Cistus monspeliensis. Int. J. Pharm. Clin. Res. 2016,8 , $243-247$.

58. Gönül, Ş.E.A.; Karapinar, M. Inhibitory effect of linden flower (Tilia flower) on the growth of foodborne pathogens. Food Microbiol. 1987, 4, 97-100. [CrossRef]

59. Yıldırım, A.; Mavi, A.; Oktay, M.; Kara, A.A.; Algur, Ö.F.; Bilaloğlu, V. Comparison of antioxidant and antimicrobial activities of Tilia (Tilia argentea Desf ex DC), sage (Salvia triloba L.), and Black tea (Camellia sinensis) extracts. J. Agric. Food Chem. 2000, 48, 5030-5034. [CrossRef]

60. Sharifa, A.A.; Neoh, Y.L.; Iswadi, M.I.; Khairul, O.; Abdul Halim, M.; Jamaludin, M.; Hing, H.L. Effects of methanol, ethanol and aqueous extract of Plantago major on gram positive bacteria, gram negative bacteria and yeast. Ann. Micr. 2008, 8, 42-44.

61. Kahyaoğlu, M.; Türkoğlu, İ. Antimicrobial activities of some plants collected in Elazı̆̆ region. Dumlupinar Univ. Bilim. Enst. Derg. 2008, 15, 1-8.

62. Ferrazzano, G.F.; Cantile, T.; Roberto, L.; Ingenito, A.; Catania, M.R.; Roscetto, E.; Pollio, A. Determination of the in vitro and in vivo antimicrobial activity on salivary Streptococci and Lactobacilli and chemical characterisation of the phenolic content of a Plantago lanceolata infusion. BioMed Res. Int. 2015, 2015, 286817. [CrossRef]

63. Metiner, K.; Ozkan, O.; Ak, S. Antibacterial effects of ethanol and acetone extract of Plantago major L. on Gram positive and Gram negative bacteria. Kafkas Univ. Vet. Fak. Derg. 2012, 18, 503-505.

64. Bonjar, S. Evaluation of antibacterial properties of some medicinal plants used in Iran. J. Ethnopharmacol. 2004, 94, 301-305. [CrossRef]

65. Valiei, M.; Shafaghat, A.; Salimi, F. Chemical composition and antimicrobial activity of the flower and root hexane extracts of Althaea officinalis in Northwest Iran. J. Med. Plants Res. 2011, 5, 6972-6976.

66. Şen, B.; Mat, A. Chemical and medicinal evaluations of the Valeriana species in Turkey. J. Fac. Pharm. İstanbul Üniv. 2015, $45,267-276$.

67. Katsarova, M.; Dimitrova, S.; Lukanov, L.; Sadakov, F.; Denev, F.; Plotnikov, E.; Kandilarov, I.; Kostadinova, I. Antioxidant activity and nontoxicity of extracts from Valeriana officinalis, Melissa officinalis, Crataegus monogyna, Hypericum perforatum, Serratula coronata and combinations Antistress 1 and Antistress 2. Bulg. Chem. Commun. 2017, 49, 93-98.

68. Lukova, P.; Karcheva-Bahchevanska, D.; Dimitrova-Dyulgerova, I.; Katsarov, P.; Mladenov, R.; Iliev, I.; Nikolova, M. A comparative pharmacognostic study and assessment of antioxidant capacity of three species from Plantago genus. Farmacia 2018, 66, 609-614. [CrossRef]

69. Wang PCh Ran, X.H.; Luo, R.H.; Ma, Q.Y.; Liu, Y.Q.; Zhoum, J.; Zhao, Y.X. Phenolic Compounds from the Roots of Valeriana officinalis var. latifolia. J. Braz. Chem. Soc. 2013, 24, 1544-1548. [CrossRef]

70. Koşar, M.; Dorman, H.J.D.; Hüsnü Can Başer, K.; Hiltunen, R. Salvia officinalis L.: Composition and Antioxidant-related Activities of a Crude Extract and Selected Sub-fractions. Nat. Prod. Commun. 2010, 9, 1453-1456. [CrossRef]

71. Seddik, K.; Dalila, B.; Saliha, D.; Saliha, D.; Smain, A.; Noureddine, C.; Abderahmane, B.; Daoud, H.; Lekhmici, A. Polyphenols and antioxidant properties of extracts from Mentha pulegium L. and Matricaria chamomilla L. Pharm. Commun. 2013, 3, 35-40. [CrossRef]

72. Ranjbar, A.; Mohsenzade, F.; Chehregani, A.; Khajavi, F.; Sifpanahi, H. Antioxidant capacity of various extracts of Matricaria Chamomilla, L. parts. Compl. Med. J. 2015, 4, 1022-1027.

73. Dimcheva, V.; Karsheva, M. Cistus incanus from Strandja Mountain as a source of bioactive antioxidants. Plants 2018, 7, 8. [CrossRef] [PubMed]

74. Lu, Y.; Foo, L.Y. Antioxidant activities of polyphenols from sage (Salvia officinalis). Food Chem. 2001, 75, 197-202. [CrossRef]

75. Koşar, M.; Dorman, H.J.D.; Baser, K.H.C.; Hiltunen, R. An improved HPLC post-column methodology for the identification of free radical scavenging phytochemicals in complex mixtures. Chromatographia 2004, 60, 635-638. [CrossRef]

76. Gericke, S.; Lübken, T.; Wolf, D.; Kaiser, M.; Hannig, C.; Speer, K. Identification of new compounds from sage flowers (Salvia officinalis L.) as markers for quality control and the influence of the manufacturing technology on the chemical composition and antibacterial activity of sage flower extracts. J. Agric. Food Chem. 2018, 66, 1843-1853. [CrossRef]

77. Redaelli, C.; Formentini, L.; Santaniello, E. PLC determination of coumarins in Matricaria chamomilla. Planta Med. 1981, 43, 412-413. [CrossRef]

78. Ohe, C.; Miami, M.; Hasegawa, C.; Ashida, K.; Sugino, M.; Kanamori, H. Seasonal variation in the production of the head and accumulation of glycosides in the head of Matricaria chamomilla. Acta Hortic. 1995, 390, 75-82. [CrossRef]

79. Gupta, V.; Mittal, P.; Bansal, P.; Khokra, S.L.; Kaushik, D. Pharmacological potential of Matricaria recutita-A review. Int J. Pharm. Sci. Drug Res. 2010, 2, 12-16. 
80. Gori, A.; Ferrini, F.; Marzano, M.A.; Tattini, M.; Centritto, M.; Baratto, M.C.; Pogni, R.; Brunetti, C. Characterisation and antioxidant activity of crude extract and polyphenolic rich fractions from C. incanus leaves. Int. J. Mol. Sci. 2016, 17, 1344. [CrossRef] [PubMed]

81. Pietta, P.; Facino, R.M.; Carini, M.; Mauri, P. Thermospray liquid chromatography mass spectrometry of flavonol glycosides from medicinal plants. J. Chrom. A 1994, 661, 121-126. [CrossRef]

82. Behrens, A.; Maie, N.; Knicker, H.; Kogel-Knabner, I. MALDI-TOF mass spectrometry and PSD fragmentation as means for the analysis of condensed tannins in plant leaves and needles. Phytochemistry 2003, 62, 1159-1170. [CrossRef]

83. Wissam, Z.; Nour, A.A.; Bushra, J.; Zein, N.; Saleh, D. Extracting and studying the antioxidant capacity of polyphenols in dry linden leaves (Tilia cordata). J. Pharm. Phytochem. 2017, 6, 258-262.

84. Kivrak, Ş.; Göktürk, T.; Kivrak, I. Determination of phenolic composition of Tilia Tomentosa flowers using UPLC-ESI-MS/MS. Int. J. Sec. Metab. 2017, 4, 249-256. [CrossRef]

85. Ashour, M.A.-G. Practical authentication and quality control of Tilia flos. Int. J. Biol. Pharm. Appl. Sci. 2017, 6, 1122-1156.

86. Gudej, J. Flavonoids, phenolic acids and coumarins from the roots of Althaea officinalis. Planta Med. 1991, 57, 284-285. [CrossRef] [PubMed]

87. Shah, S.M.A.; Akhtar, N.; Akram, M.; Shah, P.A.; Saeed, T.; Ahmed, K.; Asif, H.M. Pharmacological activity of Althaea officinalis L. J. Med. Plants Res. 2011, 5, 5662-5666. 